\title{
The Noncentral Version of the Whitney Numbers: A Comprehensive Study
}

\author{
Mahid M. Mangontarum, ${ }^{1}$ Omar I. Cauntongan, ${ }^{2}$ and Amila P. Macodi-Ringia ${ }^{1}$ \\ ${ }^{1}$ Department of Mathematics, Mindanao State University, Main Campus, 9700 Marawi City, Philippines \\ ${ }^{2}$ Department of Natural Sciences and Mathematics, Mindanao State University, Maigo School of Arts and Trades, \\ 9206 Maigo, Philippines
}

Correspondence should be addressed to Mahid M. Mangontarum; mmangontarum@yahoo.com

Received 30 December 2015; Accepted 15 March 2016

Academic Editor: Palle E. Jorgensen

Copyright (C) 2016 Mahid M. Mangontarum et al. This is an open access article distributed under the Creative Commons Attribution License, which permits unrestricted use, distribution, and reproduction in any medium, provided the original work is properly cited.

This paper is a comprehensive study of a certain generalization of Whitney-type and Stirling-type numbers which unifies the classical Whitney numbers, the translated Whitney numbers, the classical Stirling numbers, and the noncentral Stirling (or $r$ Stirling) numbers. Several identities, applications, and occurrences are also presented.

\section{Introduction}

For a finite group $G$ of order $m>0$, the Dowling lattice of rank $n$, denoted by $Q_{n}(G)$, associated with $G$ is defined by Dowling [1] as a class of geometric lattices and is known to generalize the partition lattice. Following this, Benoumhani [2] defined the Whitney numbers of the first and second kind of $Q_{n}(G)$, denoted by $w_{m}(n, k)$ and $W_{m}(n, k)$, respectively, as coefficients in the expansions of the relations

$$
\begin{aligned}
m^{n}(x)_{n} & =\sum_{k=0}^{n} w_{m}(n, k)(m x+1)^{k}, \\
(m x+1)^{n} & =\sum_{k=0}^{n} m^{k} W_{m}(n, k)(x)_{k},
\end{aligned}
$$

where

$$
(x)_{k}=x(x-1)(x-2) \cdots(x-k+1) .
$$

Fundamental properties of these numbers were mostly established by Benoumhani [2-4].

In a recent paper, Belbachir and Bousbaa [5] defined the translated Whitney numbers of the first kind $\left[\begin{array}{l}n \\ k\end{array}\right]^{(\alpha)}$ and second kind $\left\{\begin{array}{l}n \\ k\end{array}\right\}^{(\alpha)}$ to be the number of permutations (resp., partitions) of $n$ elements with $k$ cycles (resp., parts) such that the elements of each cycle (resp., parts) can mutate in $\alpha$ ways except for the dominant one. These numbers are known to obey the following relations:

$$
\begin{aligned}
(x \mid-\alpha)_{n} & =\sum_{k=0}^{n}\left[\begin{array}{l}
n \\
k
\end{array}\right]^{(\alpha)} x^{k}, \\
x^{n} & =\sum_{k=0}^{n}\left\{\begin{array}{l}
n \\
k
\end{array}\right\}^{(\alpha)}(x \mid \alpha)_{k},
\end{aligned}
$$

where

$$
(x \mid \alpha)_{n}=\prod_{i=0}^{n-1}(x-i \alpha) .
$$

Further study of these numbers was done due to Mangontarum et al. [6] and Mangontarum and Dibagulun [7].

It is important to note that the numbers in (1), (3), and (4) are known to be generalizations of the well-known Stirling 
numbers [8]. To be more precise, if $G$ is the trivial group ( $m=$ $1)$, it can be easily verified that

$$
\begin{aligned}
& w_{1}(n, k)=\left[\begin{array}{l}
n+1 \\
k+1
\end{array}\right], \\
& W_{1}(n, k)=\left\{\begin{array}{l}
n+1 \\
k+1
\end{array}\right\} .
\end{aligned}
$$

Similarly, when $\alpha=-1$ in (3) and $\alpha=1$ in (4),

$$
\begin{aligned}
& {\left[\begin{array}{l}
n \\
k
\end{array}\right]^{(-1)}=\left[\begin{array}{l}
n \\
k
\end{array}\right],} \\
& \left\{\begin{array}{l}
n \\
k
\end{array}\right\}=\left\{\begin{array}{l}
n \\
k
\end{array}\right\},
\end{aligned}
$$

where $\left[\begin{array}{l}n \\ k\end{array}\right]$ and $\left\{\begin{array}{c}n \\ k\end{array}\right\}$ denote the classical Stirling numbers of the first and second kind, respectively. Moreover, we have

$$
\begin{aligned}
& {\left[\begin{array}{l}
n \\
k
\end{array}\right]^{(\alpha)}=(-\alpha)^{n-k}\left[\begin{array}{l}
n \\
k
\end{array}\right],} \\
& \left\{\begin{array}{l}
n \\
k
\end{array}\right\}^{(\alpha)}=\alpha^{n-k}\left\{\begin{array}{l}
n \\
k
\end{array}\right\} .
\end{aligned}
$$

In line with this, Mezö [9] defined the $r$-Whitney numbers $w_{m, r}(n, k)$ and $W_{m, r}(n, k)$ of the first and second kind via expressions

$$
\begin{aligned}
m^{n}(x)_{n} & =\sum_{k=0}^{n} w_{m, r}(n, k)(m x+r)^{k}, \\
(m x+r)^{n} & =\sum_{k=0}^{n} m^{k} W_{m, r}(n, k)(x)_{k},
\end{aligned}
$$

respectively, to obtain remarkable formulas related to the Bernoulli and Harmonic polynomials. Notice that by suitable assignments of parameters, the previously mentioned numbers appear to be particular cases of (9) and (10). Further combinatorial and algebraic properties of these numbers were later on studied by Cheon and Jung [10] and Mezö and Ramirez [11].

The motivation of this paper is partly influenced by the pair of generalizations of the numbers $\left[\begin{array}{l}n \\ k\end{array}\right]$ and $\left\{\begin{array}{l}n \\ k\end{array}\right\}$ earlier considered by Koutras [12] which were defined by the relations

$$
\begin{aligned}
(t)_{n} & =\sum_{k=0}^{n} \frac{1}{k !}\left[\frac{d^{k}}{d t^{k}}(t)_{n}\right]_{t=a}(t-a)^{k}, \\
(t-a)^{n} & =\sum_{k=0}^{n} \frac{1}{k !}\left[\Delta^{k}(t-a)^{n}\right]_{t=0}(t)_{k},
\end{aligned}
$$

where the following notations are used:

$$
s_{a}(n, k)=(1 / k !)\left[\left(d^{k} / d t^{k}\right)(t)_{n}\right]_{t=a}:=\text { noncentral }
$$

Stirling numbers of the first kind,

$$
S_{a}(n, k)=(1 / k !)\left[\Delta^{k}(t-a)^{n}\right]_{t=0}:=\text { noncentral Stirling }
$$
numbers of the second kind.

Note that these numbers can be shown to be equivalent to Broder's [13] $r$-Stirling numbers. However, the methods by which the former were defined appear to be of distinct motivation (cf. [13, equations (3) and (4)]). Keeping this in mind, we propose "noncentral" versions for the classical Whitney numbers parallel to the work of Koutras as seen in (11) and (12). These will serve as unified generalizations of all the abovementioned sequences of special numbers. In this comprehensive study, we present fundamental combinatorial properties such as recurrence relations, generating functions and explicit formulas, and derive more results such as the orthogonality and the inverse relations, matrix decompositions, Hankel transform, and other notable identities. Several conjectures and questions are also mentioned for further research.

\section{Definitions and Basic Properties}

2.1. Noncentral Whitney Numbers of the First Kind. For any real numbers $a, t$, nonnegative integer $n$, and positive integer $m$, the expansion of $(t \mid m)_{n}$ in a Taylor series gives

$$
(t \mid m)_{n}=\sum_{k=0}^{n} \frac{1}{k !}\left[\frac{d^{k}}{d t^{k}}(t \mid m)_{n}\right]_{t=a}(t-a)^{k} .
$$

We define the noncentral Whitney numbers of the first kind, denoted by $\widetilde{w}_{m, a}(n, k)$, as

$$
\widetilde{w}_{m, a}(n, k)=\frac{1}{k !}\left[\frac{d^{k}}{d t^{k}}(t \mid m)_{n}\right]_{t=a}
$$

with the initial conditions $\widetilde{w}_{m, a}(0,0)=1$ and $\widetilde{w}_{m, a}(n, k)=0$ if $n<k$ or $n, k<0$. Obviously, we have the next identity.

Proposition 1. The numbers $\widetilde{w}_{m, a}(n, k)$ satisfy the horizontal generating function

$$
(t \mid m)_{n}=\sum_{k=0}^{n} \widetilde{w}_{m, a}(n, k)(t-a)^{k} .
$$

Notice that (15) is equivalent to

$$
m^{n}(x)_{n}=\sum_{k=0}^{n} \widetilde{w}_{m, a}(n, k)(m x-a)^{k}
$$

when $t$ is replaced with $m x$. Hence, we can see that from (9) and (11)

$$
\begin{gathered}
\widetilde{w}_{m,-r}(n, k)=w_{m, r}(n, k), \\
\widetilde{w}_{1, a}(n, k)=s_{a}(n, k) .
\end{gathered}
$$

Moreover,

$$
\begin{aligned}
& \widetilde{w}_{-\alpha, 0}(n, k)=\left[\begin{array}{l}
n \\
k
\end{array}\right]^{(\alpha)}, \\
& \widetilde{w}_{1,0}(n, k)=\left[\begin{array}{l}
n \\
k
\end{array}\right] .
\end{aligned}
$$


From (15), one has

$$
\begin{aligned}
& \sum_{k=0}^{n+1} \widetilde{w}_{m, a}(n+1, k)(m x-a)^{k}=\sum_{k=0}^{n} \widetilde{w}_{m, a}(n, k) \\
& \cdot(m x-a)^{k}(m x-n m) \\
& =\sum_{k=0}^{n+1}\left\{\widetilde{w}_{m, a}(n, k-1)+(a-n m) \widetilde{w}_{m, a}(n, k)\right\} \\
& \cdot(m x-a)^{k} .
\end{aligned}
$$

Comparing coefficients of $(m x-a)^{k}$ yields the next identity useful in finding the values of $\widetilde{w}_{m, a}(n, k)$.

Proposition 2. The noncentral Whitney numbers of the first kind satisfy the following triangular recurrence relation:

$$
\begin{aligned}
\widetilde{w}_{m, a}(n+1, k)= & \widetilde{w}_{m, a}(n, k-1) \\
& +(a-n m) \widetilde{w}_{m, a}(n, k) .
\end{aligned}
$$

It can be seen from this recurrence relation that

$$
\begin{aligned}
& \widetilde{w}_{m, a}(n, 0)=(a \mid m)_{n}, \\
& \widetilde{w}_{m, a}(n, k)=1, \quad k=n .
\end{aligned}
$$

The next corollary can be obtained by successive application of (20).

Corollary 3. The noncentral Whitney numbers of the first kind satisfy the following recurrence relations:

$$
\begin{aligned}
& \widetilde{w}_{m, a}(n+1, k+1)=\sum_{j=k}^{n} \frac{(a \mid m)_{n+1}}{(a \mid m)_{j+1}} \widetilde{w}_{m, a}(j, k), \\
& \widetilde{w}_{m, a}(n, k)=\sum_{j=0}^{n-k}(m n-a)^{j} \widetilde{w}_{m, a}(n+1, k+j+1) .
\end{aligned}
$$

Note that (15) can be written as

$$
m^{n} n !\left(\begin{array}{c}
\frac{x+a}{m} \\
n
\end{array}\right)=\sum_{k=0}^{n} \widetilde{w}_{m, a}(n, k) x^{k}
$$

when $x$ is replaced with $x+a$. We are now ready to state the following proposition.

Proposition 4. The exponential generating function of the sequence $\left\{\widetilde{w}_{m, a}(n, k)\right\}$ is given by

$$
\sum_{n=k}^{\infty} \widetilde{w}_{m, a}(n, k) \frac{z^{n}}{n !}=(1+m z)^{a / m} \frac{[\log (1+m z)]^{k}}{m^{k} k !} .
$$

Proof. Multiplying both sides of (24) by $z^{n} / n$ ! and summing over $n$ gives us

$$
\sum_{n=0}^{\infty}\left(\begin{array}{c}
\frac{x+a}{m} \\
n
\end{array}\right) m^{n} z^{n}=\sum_{n=0}^{\infty} \sum_{k=0}^{n} \widetilde{w}_{m, a}(n, k) x^{k} \frac{z^{n}}{n !} .
$$

Since the left-hand side is just

$$
\begin{aligned}
& \sum_{n=0}^{(x+a) / m}\left(\begin{array}{c}
\frac{x+a}{m} \\
n
\end{array}\right)(m z)^{n} \\
& =(1+m z)^{a / m} \sum_{k=0}^{\infty}\left(\frac{x}{m}\right) \frac{[\log (1+m z)]^{k}}{k !},
\end{aligned}
$$

then we have

$$
\begin{aligned}
\sum_{k=0}^{\infty}\left\{\sum_{n=k}^{\infty} \widetilde{w}_{m, a}(n, k) \frac{z^{n}}{n !}\right\} x^{k} \\
=\sum_{k=0}^{\infty}\left\{\frac{(1+m z)^{a / m}}{k !} \cdot\left[\frac{\log (1+m z)}{m}\right]^{k}\right\} x^{k} .
\end{aligned}
$$

Comparing the coefficients of $x^{k}$ completes the proof.

Theorem 5. The noncentral Whitney numbers $\widetilde{w}_{m, a}(n, k)$ satisfy the following relations:

$$
\begin{aligned}
& \widetilde{w}_{m, a}(n, k)=\sum_{i=k}^{n}\left(\begin{array}{l}
i \\
k
\end{array}\right) a^{i-k} m^{n-i}\left[\begin{array}{l}
n \\
i
\end{array}\right], \\
& \widetilde{w}_{m, a}(n, k)=\sum_{i=k}^{n}\left(\begin{array}{l}
i \\
k
\end{array}\right) a^{i-k}\left[\begin{array}{l}
n \\
i
\end{array}\right]^{(m)} .
\end{aligned}
$$

Proof. Notice that (30) is an obvious consequence of (29). Hence, we only choose to prove (29). Note that multiplying by $m^{n}$ the defining relation for the Stirling numbers $\left[\begin{array}{l}n \\ k\end{array}\right]$ given by

$$
(x)_{n}=\sum_{i=0}^{n}\left[\begin{array}{l}
n \\
i
\end{array}\right] x^{i}
$$

yields

$$
\begin{aligned}
m^{n}(x)_{n} & =m^{n} \sum_{i=0}^{n}\left[\begin{array}{l}
n \\
i
\end{array}\right]\left(\frac{m x-a+a}{m}\right)^{i} \\
& =m^{n} \sum_{i=0}^{n}\left[\begin{array}{l}
n \\
i
\end{array}\right] \sum_{k=0}^{i}\left(\begin{array}{l}
i \\
k
\end{array}\right) a^{i-k}(m x-a)^{k} \frac{1}{m^{i}} \\
& =\sum_{k=0}^{n}\left\{\sum_{i=k}^{n} m^{n-i}\left[\begin{array}{l}
n \\
i
\end{array}\right]\left(\begin{array}{l}
i \\
k
\end{array}\right) a^{i-k}\right\}(m x-a)^{k} .
\end{aligned}
$$

Comparing the coefficients of $(m x-a)^{k}$ with (16) gives the desired result.

It is known that there is no simple method in expressing first kind Stirling-type numbers explicitly. In the next theorem, we express the numbers $\widetilde{w}_{m, a}(n, k)$ in elementary symmetric polynomial form by induction.

Theorem 6. The numbers $\widetilde{w}_{m, a}(n, k)$ satisfy the explicit formula

$$
\widetilde{w}_{m, a}(n, k)=\sum_{0 \leq i_{1}<i_{2}<\cdots<i_{n-k} \leq n-1} \prod_{j=1}^{n-k}\left(a-i_{j} m\right) .
$$


Proof. Note that the theorem yields $\widetilde{w}_{m, a}(0,0)=1$, which is in line with the initial value of $\widetilde{w}_{m, a}(n, k)$ stated earlier in this section. Now, suppose the theorem holds up to $n$ for $k=0,1,2, \ldots, n$. Then by (20),

$$
\begin{aligned}
& \widetilde{w}_{m, a}(n+1, k) \\
& =\sum_{0 \leq i_{1}<i_{2}<\cdots<i_{n+1-k} \leq n-1} \prod_{j=1}^{n+1-k}\left(a-i_{j} m\right) \\
& \quad+(a-n m) \sum_{0 \leq i_{1}<i_{2}<\cdots<i_{n-k} \leq n-1} \prod_{j=1}^{n-k}\left(a-i_{j} m\right) \\
& =\sum_{0 \leq i_{1}<i_{2}<\cdots<i_{n+1-k} \leq n} \prod_{j=1}^{n+1-k}\left(a-i_{j} m\right) .
\end{aligned}
$$

Finally (33) yields $\widetilde{w}_{m, a}(n+1, k)=1$ when $k=n+1$. This is in accordance with (22).

2.2. Noncentral Whitney Numbers of the Second Kind. Analogous to what is being done in (12), we define the noncentral Whitney numbers of the second kind by

$$
m^{k} k ! \widetilde{W}_{m, a}(n, k)=\left[\Delta^{k}(m t-a)^{n}\right]_{t=0},
$$

for any real numbers $a$ and $t$, nonnegative integer $n$, and positive integer $m$. Now, let $f(x)=(m x-a)^{n}$. The known difference operator

$$
\Delta^{k} f(x)=\sum_{j=0}^{k}(-1)^{k-j}\left(\begin{array}{l}
k \\
j
\end{array}\right) f(x+j)
$$

yields the explicit formula

$$
\left[\Delta^{k}(m x-a)^{n}\right]_{x=0}=\sum_{j=0}^{k}(-1)^{k-j}\left(\begin{array}{l}
k \\
j
\end{array}\right)(m j-a)^{n} .
$$

Hence we propose the following combinatorial properties of the numbers $\widetilde{W}_{m, a}(n, k)$.

Proposition 7. An explicit formula for $\widetilde{W}_{m, a}(n, k)$ is given by

$$
\widetilde{W}_{m, a}(n, k)=\frac{1}{m^{k} k !} \sum_{j=0}^{k}(-1)^{k-j}\left(\begin{array}{l}
k \\
j
\end{array}\right)(m j-a)^{n} .
$$

Moreover, the exponential generating function of the sequence $\left\{\widetilde{W}_{m, a}(n, k)\right\}$ is given by

$$
\sum_{n=k}^{\infty} \widetilde{W}_{m, a}(n, k) \frac{z^{n}}{n !}=\frac{e^{-a z}}{m^{k} k !}\left(e^{m z}-1\right)^{k}
$$

while the horizontal generating function is

$$
(t-a)^{n}=\sum_{k=0}^{n} \widetilde{W}_{m, a}(n, k)(t \mid m)_{k} .
$$

Replacing $t$ with $m x$ in (40) yields

$$
(m x-a)^{k}=\sum_{k=0}^{n} m^{k} \widetilde{W}_{m, a}(n, k)(x)_{k} .
$$

Hence,

$$
\begin{aligned}
& \widetilde{W}_{m,-r}(n, k)=W_{m, r}(n, k), \\
& \widetilde{W}_{1, a}(n, k)=S_{a}(n, k), \\
& \widetilde{W}_{\alpha, 0}(n, k)=\left\{\begin{array}{l}
n \\
k
\end{array}\right\}^{(\alpha)}, \\
& \widetilde{W}_{1,0}(n, k)=\left\{\begin{array}{l}
n \\
k
\end{array}\right\} .
\end{aligned}
$$

The next identity is also immediately obtained.

Proposition 8. The noncentral Whitney numbers of the second kind satisfy the following triangular recurrence relation:

$$
\begin{aligned}
\widetilde{W}_{m, a}(n+1, k)= & \widetilde{W}_{m, a}(n, k-1) \\
& +(k m-a) \widetilde{W}_{m, a}(n, k) .
\end{aligned}
$$

Obviously,

$$
\begin{aligned}
& \widetilde{W}_{m, a}(n, 0)=(-a)^{n}, \\
& \widetilde{W}_{m, a}(n, k)=1
\end{aligned}
$$

when $k=n$. Also, from (23), we deduce the following.

Corollary 9. The noncentral Whitney numbers of the second kind satisfy the following recurrence relations:

$$
\begin{aligned}
& \widetilde{W}_{m, a}(n+1, k+1) \\
& =\sum_{j=k}^{n}[m(k+1)-a]^{n-j} \widetilde{W}_{m, a}(j, k) ; \\
& \widetilde{W}_{m, a}(n, k) \\
& =\sum_{j=0}^{n-k}(-1)^{j} \frac{(-a \mid m)_{n+1}}{(-a \mid m)_{n-j+1}} \widetilde{W}_{m, a}(n+1, k+j+1) .
\end{aligned}
$$

It is also possible to express $\widetilde{W}_{m, a}(n, k)$ in terms of the classical Stirling numbers of the second kind. To do so, note that

$$
(m x-a)^{n}=\sum_{j=0}^{n}\left(\begin{array}{l}
n \\
j
\end{array}\right)(-a)^{n-j} m^{j} x^{j} .
$$

Using the defining relation

$$
x^{n}=\sum_{k=0}^{n}\left\{\begin{array}{l}
n \\
k
\end{array}\right\}(x)_{k}
$$


of the Stirling numbers of the second kind, we obtain

$$
\begin{aligned}
(m x-a)^{n} & =\sum_{j=0}^{n}\left(\begin{array}{l}
n \\
j
\end{array}\right)(-a)^{n-j} m^{j} \sum_{k=0}^{j}\left\{\begin{array}{l}
j \\
k
\end{array}\right\}(x)_{k} \\
& =\sum_{k=0}^{n}\left\{\sum_{j=k}^{n}\left(\begin{array}{l}
n \\
j
\end{array}\right)(-a)^{n-j} m^{j}\left\{\begin{array}{l}
j \\
k
\end{array}\right\}\right\}(x)_{k} .
\end{aligned}
$$

Comparing the coefficients of $(x)_{k}$ with (40) yields

$$
\widetilde{W}_{m, a}(n, k)=\sum_{j=k}^{n}\left(\begin{array}{l}
n \\
j
\end{array}\right)(-a)^{n-j} m^{j-k}\left\{\begin{array}{l}
j \\
k
\end{array}\right\} .
$$

Let us formally state this in the next theorem.

Theorem 10. The numbers $\widetilde{W}_{m, a}(n, k)$ satisfy the following identities:

$$
\begin{aligned}
& \widetilde{W}_{m, a}(n, k)=\sum_{j=k}^{n}\left(\begin{array}{l}
n \\
j
\end{array}\right)(-a)^{n-j} m^{j-k}\left\{\begin{array}{l}
j \\
k
\end{array}\right\}, \\
& \widetilde{W}_{m, a}(n, k)=\sum_{j=k}^{n}\left(\begin{array}{l}
n \\
j
\end{array}\right)(-a)^{n-j}\left\{\begin{array}{l}
j \\
k
\end{array}\right\} .
\end{aligned}
$$

Proof. The other equality follows directly.

The next theorem can be proved by similar method used to prove (33).

Theorem 11. The numbers $\widetilde{W}_{m, a}(n, k)$ satisfy the explicit formula in complete symmetric polynomial form given by

$$
\widetilde{W}_{m, a}(n, k)=(-1)^{n-k} \sum_{0 \leq i_{1} \leq i_{2} \leq \cdots \leq i_{n-k} \leq k} \prod_{j=1}^{n-k}\left(a-i_{j} m\right) .
$$

2.3. Application to the Bernoulli Polynomials. The wellknown Bernoulli polynomials $B_{n}(x)$ defined by the exponential generating function [9]

$$
\sum_{n=0}^{\infty} B_{n}(x) \frac{z^{n}}{n !}=\frac{z e^{z x}}{e^{z}-1}
$$

where $B_{n}(0)=B_{n}$ are the Bernoulli numbers. In relation to this, Mezö [9] obtained some identities showing interesting relationships between the $r$-Whitney numbers and the Bernoulli polynomials. The said identities are as follows:

$$
\begin{aligned}
& \left(\begin{array}{c}
n+1 \\
l
\end{array}\right) B_{n-l+1} \\
& =\frac{n+1}{m^{n-l+1}} \sum_{k=0}^{n} W_{m, r}(n, k) \frac{w_{m, r}(k+1, l)}{k+1}, \\
& \left(\begin{array}{c}
n+1 \\
l
\end{array}\right) B_{n-l+1}\left(\frac{r}{m}\right) \\
& =\frac{n+1}{m^{n}} \sum_{k=0}^{n} \frac{m^{k}}{k+1} W_{m, r}(n, k)\left[\begin{array}{c}
k+1 \\
l
\end{array}\right] .
\end{aligned}
$$

Note that when $m=1$ and $r=0$ in both (54) and (55), we obtain the classical identity [14]

$$
\left(\begin{array}{c}
n+1 \\
l
\end{array}\right) B_{n-l+1}=(n+1) \sum_{k=0}^{n}\left\{\begin{array}{l}
n \\
k
\end{array}\right\}\left[\begin{array}{c}
k+1 \\
l
\end{array}\right] \frac{1}{k+1} .
$$

Following the same method used by Mezö [9] and through the aid of the exponential generating function in (25) and the identity

$$
\sum_{n=k}^{\infty}\left[\begin{array}{l}
n \\
k
\end{array}\right] \frac{z^{n}}{n !}=\frac{[\log (1+z)]^{l}}{l !}
$$

we propose an analogous relationship between the Bernoulli polynomials and the noncentral Whitney numbers of both kinds as follows.

Proposition 12. The noncentral translated Whitney numbers $\widetilde{W}_{m, a}(n, k)$ and $\widetilde{w}_{m, a}(k+1, l)$ satisfy the following identities:

$$
\begin{aligned}
& \left(\begin{array}{c}
n+1 \\
l
\end{array}\right) B_{n-l+1} \\
& =\frac{n+1}{m^{n-l+1}} \sum_{k=0}^{n} \widetilde{W}_{m, a}(n, k) \frac{\widetilde{w}_{m, a}(k+1, l)}{k+1}, \\
& \left(\begin{array}{c}
n+1 \\
l
\end{array}\right) B_{n-l+1}\left(-\frac{a}{m}\right) \\
& =\frac{n+1}{m^{n}} \sum_{k=0}^{n} \frac{m^{k}}{k+1} \widetilde{W}_{m, a}(n, k)\left[\begin{array}{c}
k+1 \\
l
\end{array}\right] .
\end{aligned}
$$

\section{Matrix Relations for the Noncentral Whitney Numbers}

\subsection{Orthogonality and Inverse Relations}

Proposition 13. The noncentral Whitney numbers of the first and second kind satisfy the following orthogonality relations:

$$
\begin{aligned}
& \sum_{k=j}^{n} \widetilde{W}_{m, a}(n, k) \widetilde{w}_{m, a}(k, j)=\sum_{k=j}^{n} \widetilde{w}_{m, a}(n, k) \widetilde{W}_{m, a}(k, j) \\
& \quad=\delta_{n j},
\end{aligned}
$$

where

$$
\delta_{n j}= \begin{cases}0, & \text { if } j \neq n \\ 1, & \text { if } j=n\end{cases}
$$

is the Kronecker delta.

This proposition can be easily proved by combining (16) and (40). 
Now, since $\widetilde{W}_{m, a}(n, k)=\widetilde{w}_{m, a}(n, k)=0$ when $n<k$, then we have

$$
\begin{aligned}
& \sum_{k=0}^{\infty} \widetilde{W}_{m, a}(n, k) \widetilde{w}_{m, a}(k, j)=\sum_{k=0}^{\infty} \widetilde{w}_{m, a}(n, k) \widetilde{W}_{m, a}(k, j) \\
& =\delta_{n j} .
\end{aligned}
$$

If we define $\mathcal{N}_{m, a}=\left(\widetilde{W}_{m, a}(i, l)\right)$ to be the infinite matrix with $\widetilde{W}_{m, a}(i, l)$ as the $(i, l)$ th entries for $i, l=0,1,2,3, \ldots$ and $\mathscr{M}_{m, a}=\left(\widetilde{w}_{m, a}(i, l)\right)$ as similar matrix for $\widetilde{w}_{m, a}(i, l)$ then we have

$$
\mathcal{N}_{m, a} \cdot \mathscr{M}_{m, a}=\mathscr{M}_{m, a} \cdot \mathcal{N}_{m, a}=\mathscr{F},
$$

where $\mathscr{I}$ is the infinite-dimensional identity matrix. Thus, $\mathscr{M}_{m, a}=\mathcal{N}_{m, a}^{-1}$, where $\mathcal{N}_{m, a}^{-1}$ is the inverse of $\mathcal{N}_{m, a}$.

Corollary 14. The following inverse relations hold:

$$
\begin{aligned}
f_{n} & =\sum_{k=0}^{n} \widetilde{w}_{m, a}(n, k) g_{k} \Longleftrightarrow \\
g_{n} & =\sum_{k=0}^{n} \widetilde{W}_{m, a}(n, k) f_{k}, \\
f_{k} & =\sum_{n=k}^{\infty} \widetilde{w}_{m, a}(n, k) g_{n} \Longleftrightarrow \\
g_{k} & =\sum_{n=k}^{\infty} \widetilde{W}_{m, a}(n, k) f_{n} .
\end{aligned}
$$

3.2. Matrix Decomposition of the Noncentral Whitney Numbers. In a recent paper, Pan [15] introduced a remarkable matrix decomposition to give an explicit and nonrecursive way of computing the Unified Generalized Stirling numbers of Hsu and Shiue [16]. The said result is as follows [15, Theorem 7]:

$$
\mathcal{S}_{\alpha, \beta, \gamma}=\mathcal{S}_{\alpha, 0,0} \cdot \mathcal{S}_{0,0, \gamma} \cdot \mathcal{S}_{0, \beta, 0} .
$$

Here, the matrix $\mathcal{S}_{\alpha, \beta, \gamma}$ is given by

$$
\mathcal{S}_{\alpha, \beta, \gamma}=(S(n, k ; \alpha, \beta, \gamma)) \text {, }
$$

where $S(n, k ; \alpha, \beta, \gamma)$ is Hsu and Shiue's [16] generalized Stirling numbers defined by

$$
(x \mid \alpha)_{n}=\sum_{k=0}^{\infty} S(n, k ; \alpha, \beta, \gamma)(x-\gamma \mid \beta)_{k} .
$$

It can be verified that the $(n, k)$ th entries of the matrices $\mathcal{S}_{\alpha, 0,0}$, $\mathcal{S}_{0, \beta, 0}$, and $\mathcal{S}_{0,0, \gamma}$ are

$$
\begin{gathered}
\alpha^{n-k}\left[\begin{array}{l}
n \\
k
\end{array}\right], \\
\beta^{n-k}\left\{\begin{array}{l}
n \\
k
\end{array}\right\}, \\
\gamma^{n-k}\left(\begin{array}{l}
n \\
k
\end{array}\right),
\end{gathered}
$$

respectively.
Although the noncentral Whitney numbers can be written as

$$
\begin{aligned}
& S(n, k ;-a, m, 0)=\widetilde{w}_{m, a}(n, k), \\
& S(n, k ; 0, m,-a)=\widetilde{W}_{m, a}(n, k),
\end{aligned}
$$

it is not wise to assume that

$$
\begin{aligned}
& \mathscr{M}_{m, a}:=\mathcal{S}_{-a, m, 0}=\mathcal{S}_{-a, 0,0} \cdot \mathcal{S}_{0,0,0} \cdot \mathcal{S}_{0, m, 0}, \\
& \mathcal{N}_{m, a}:=\mathcal{S}_{0, m, m}=\mathcal{S}_{0,0,0} \cdot \mathcal{S}_{0,0,-a} \cdot \mathcal{S}_{0, m, 0} .
\end{aligned}
$$

Hence, it is justifiable to establish the matrix decomposition for the noncentral Whitney numbers of both kinds. For convenience, we will refer to the matrices $\mathscr{M}_{m, a}$ and $\mathcal{N}_{m, a}$ as noncentral Whitney matrix of the first and second kind, respectively. Also, we let

$$
\mathscr{V}_{\alpha}(x)=\left(1, x,(x \mid \alpha)_{2},(x \mid \alpha)_{3}, \ldots,(x \mid \alpha)_{n}, \ldots\right)^{T}
$$

be an infinite column vector.

Remark 15. In reference to the relations in (15) and (40), the following identities seem natural:

$$
\begin{aligned}
\mathscr{V}_{m}(x) & =\mathscr{M}_{m, a} \cdot \mathscr{V}_{0}(x-a), \\
\mathscr{V}_{0}(x-a) & =\mathscr{N}_{m, a} \cdot \mathscr{V}_{m}(x) .
\end{aligned}
$$

Since $\widetilde{w}_{m, 0}(n, k)=\left[\begin{array}{l}n \\ k\end{array}\right]^{(-m)}$ and $\widetilde{W}_{m, 0}(n, k)=\left\{\begin{array}{l}n \\ k\end{array}\right\}^{(m)}$, then we have the following matrices:

$$
\begin{aligned}
\mathscr{M}_{m, 0} & =\left(\left[\begin{array}{l}
n \\
k
\end{array}\right]^{(-m)}\right), \\
\mathcal{N}_{m, 0} & =\left(\left\{\begin{array}{l}
n \\
k
\end{array}\right\}^{(m)}\right) .
\end{aligned}
$$

By using the "signed" translated Whitney numbers $w_{(m)}^{*}(n, k)$ [7], the matrix $\mathscr{M}_{m, 0}$ can also be rewritten as

$$
\mathscr{M}_{m, 0}=\left(w_{(m)}^{*}(n, k)\right) .
$$

Now, from (15),

$$
0=\sum_{k=0}^{n} \widetilde{w}_{0, a}(n, k)(-a)^{k}
$$

while the binomial theorem yields

$$
0=(-a+a)^{n}=\sum_{k=0}^{n}\left(\begin{array}{l}
n \\
k
\end{array}\right) a^{n-k}(-a)^{k} .
$$

Thus, we have

$$
\widetilde{w}_{0, a}(n, k)=a^{n-k}\left(\begin{array}{l}
n \\
k
\end{array}\right) .
$$


It can be shown using a similar manner that

$$
\widetilde{W}_{0, a}(n, k)=(-a)^{n-k}\left(\begin{array}{l}
n \\
k
\end{array}\right) .
$$

It then follows that

$$
\begin{aligned}
& \mathscr{M}_{0, a}=\left(a^{n-k}\left(\begin{array}{l}
n \\
k
\end{array}\right)\right), \\
& \mathcal{N}_{0, a}=\left((-a)^{n-k}\left(\begin{array}{l}
n \\
k
\end{array}\right)\right) .
\end{aligned}
$$

We are now ready to state the next theorem.

Theorem 16. The decomposition formulas of the matrices $\mathscr{M}_{m, a}$ and $\mathcal{N}_{m, a}$ are

$$
\begin{aligned}
& \mathscr{M}_{m, a}=\mathscr{M}_{m, 0} \cdot \mathscr{M}_{0, a}, \\
& \mathcal{N}_{m, a}=\mathcal{N}_{0, a} \cdot \mathcal{N}_{m, 0} .
\end{aligned}
$$

Proof. Since

$$
\begin{aligned}
& \mathscr{V}_{m}(x)=\mathscr{M}_{m, 0} \cdot \mathscr{V}_{0}(x), \\
& \mathscr{V}_{0}(x)=\mathscr{M}_{0, a} \cdot \mathscr{V}_{0}(x-a)
\end{aligned}
$$

then

$$
\mathscr{V}_{m}(x)=\mathscr{M}_{m, 0} \mathscr{M}_{0, a} \cdot \mathscr{V}_{0}(x-a) .
$$

Combining this with (71) gives us

$$
\left(\mathscr{M}_{m, a}-\mathscr{M}_{m, 0} \cdot \mathscr{M}_{0, a}\right) \mathscr{V}_{0}(x-a)=\mathbf{0}
$$

where $\mathbf{0}$ denotes an infinite-dimensional zero matrix. Consequently, because $x$ is an arbitrary real or complex number and $\mathscr{V}_{0}(x-a)$ is a nonzero vector, then

$$
\mathscr{M}_{m, a}=\mathscr{M}_{m, 0} \cdot \mathscr{M}_{0, a} .
$$

Equation (81) can be shown similarly.

\section{Noncentral Dowling and Noncentral Tanny-Dowling Polynomials}

Benoumhani $[2,3]$ was the first to introduce the following familiar polynomials:

$$
\begin{aligned}
& D_{m}(n ; x)=\sum_{k=0}^{n} W_{m}(n, k) x^{k}, \\
& \mathscr{F}_{m}(n ; x)=\sum_{k=0}^{n} k ! W_{m}(n, k) x^{k} .
\end{aligned}
$$

$D_{m}(n ; x)$ and $\mathscr{F}_{m}(n ; x)$ are known as the Dowling and the Tanny-Dowling polynomials. Moreover, when $m=1$ in (87), the resulting polynomial

$$
\mathscr{F}_{1}(n ; x)=\sum_{k=0}^{n} k !\left\{\begin{array}{l}
n \\
k
\end{array}\right\} x^{k}
$$

is called geometric polynomials [17] and was earlier studied by Tanny [18].

Denoted by $\widetilde{\mathscr{D}}_{m, a}(n ; x)$ and $\widetilde{\mathscr{F}}_{m, a}(n ; x)$, the noncentral Dowling and the noncentral Tanny-Dowling polynomials can be defined as

$$
\begin{aligned}
& \widetilde{\mathscr{D}}_{m, a}(n ; x)=\sum_{k=0}^{n} \widetilde{W}_{m, a}(n, k) x^{k}, \\
& \widetilde{\mathscr{F}}_{m, a}(n ; x)=\sum_{k=0}^{n} k ! \widetilde{W}_{m, a}(n, k) x^{k} .
\end{aligned}
$$

For brevity, we also call $\widetilde{\mathscr{D}}_{m, a}(n ; 1) \equiv \widetilde{\mathscr{D}}_{m, a}(n)$ and $\widetilde{\mathscr{F}}_{m, a}(n$; $1) \equiv \widetilde{\mathscr{F}}_{m, a}(n)$ as the noncentral Dowling and the noncentral Tanny-Dowling numbers, respectively. Notice that through the use of the exponential generating function in (39) and the explicit formula (38), the noncentral Dowling polynomials can be defined alternatively by (91) or explicitly by the Dobinski-type identity (92) in the following proposition.

Proposition 17. The following identities hold:

$$
\begin{aligned}
\sum_{n=0}^{\infty} \widetilde{\mathscr{D}}_{m, a}(n ; x) \frac{z^{n}}{n !} & =e^{-a z+\left(e^{m z}-1\right)(x / m)}, \\
\widetilde{\mathscr{D}}_{m, a}(n ; x) & =e^{-x / m} \sum_{i=0}^{\infty}\left(\frac{x}{m}\right)^{i} \frac{(m i-a)^{n}}{i !} .
\end{aligned}
$$

These identities are actually equivalent to those of the $r$ Whitney polynomials when $a=-r$ and are generalizations of the translated Dowling polynomials (cf. [6, equations (22) and (25)]). As for the noncentral Tanny-Dowling polynomials, since (39) can be rewritten as

$$
\sum_{n=k}^{\infty} k ! \widetilde{W}_{m, a}(n, k) \frac{z^{n}}{n !}=e^{-a z}\left(\frac{e^{m z}-1}{m}\right)^{k},
$$

then we get

$$
\begin{aligned}
\sum_{n=k}^{\infty} \widetilde{\mathscr{F}}_{m, a}(n ; x) \frac{z^{n}}{n !} & =\sum_{n=0}^{\infty} \sum_{k=0}^{n} k ! \widetilde{W}_{m, a}(n, k) \frac{z^{n}}{n !} \\
& =\sum_{k=0}^{\infty} e^{-a z}\left(\frac{e^{m z}-1}{m}\right)^{k} \\
& =e^{-a z}\left(\frac{1}{1-\left(e^{m z}-1\right) x / m}\right) .
\end{aligned}
$$

This is equivalent to (95) in the next theorem.

Theorem 18. The polynomials $\widetilde{\mathscr{F}}_{m, a}(n ; x)$ satisfy the exponential generating function

$$
\sum_{n=k}^{\infty} \widetilde{\mathscr{F}}_{m, a}(n ; x) \frac{z^{n}}{n !}=\frac{m e^{-a z}}{m-x\left(e^{m z}-1\right)} .
$$

The case in (95), where $x=m y$, immediately yields

$$
\sum_{n=k}^{\infty} \widetilde{\mathscr{F}}_{m, a}(n ; m y) \frac{z^{n}}{n !}=\frac{e^{-a z}}{1-y\left(e^{m z}-1\right)} .
$$


Equations (95) and (96) reduce to Benoumhani's results (cf. [3, Theorem 3]) when $a=-1$. The next theorem presents the explicit formula for the polynomials $\widetilde{\mathscr{F}}_{m, a}(n ; x)$.

Theorem 19. The following explicit formula holds:

$$
\widetilde{\mathscr{F}}_{m, a}(n ; x)=\frac{m}{x+m} \sum_{k=0}^{\infty}\left(\frac{x}{x+m}\right)^{k}(m k-a)^{n} .
$$

Proof. To prove this theorem, we first show that

$$
\widetilde{\mathscr{F}}_{m, a}(n ; m y)=\frac{1}{1+y} \sum_{k=0}^{\infty}\left(\frac{y}{1+y}\right)^{k}(m k-a)^{n} .
$$

Note that by algebraic manipulation, one readily gets

$$
\begin{aligned}
& \sum_{n=0}^{\infty}\left(\frac{1}{1+y} \sum_{k=0}^{\infty}\left(\frac{y}{1+y}\right)^{k}(m k-a)^{n}\right) \frac{z^{n}}{n !} \\
& \quad=\sum_{n=0}^{\infty}\left(\frac{1}{1+y}\right. \\
& \left.\cdot \sum_{k=0}^{\infty}\left(\frac{y}{1+y}\right)^{k} \cdot \sum_{i=0}^{n}\left(\begin{array}{c}
n \\
i
\end{array}\right)(k m)^{n-i}(-a)^{i}\right) \frac{z^{n}}{n !} \\
& =\frac{1}{1+y} \sum_{i=0}^{\infty} \frac{(-a z)^{i}}{i !} \sum_{k=0}^{\infty}\left(\frac{y}{1+y}\right)^{k} \cdot \sum_{n=i}^{\infty} \frac{(k m)^{n-i}}{(n-i) !} z^{n-i} .
\end{aligned}
$$

Reindexing the third sum and using (96), we get

$$
\begin{aligned}
& \frac{1}{1+y} \sum_{i=0}^{\infty} \frac{(-a z)^{i}}{i !} \sum_{k=0}^{\infty}\left(\frac{y}{1+y}\right)^{k} \sum_{n=i}^{\infty} \frac{(k m z)^{n-i}}{(n-i) !} \\
& =\frac{1}{1+y} e^{-a z} \sum_{k=0}^{\infty}\left(\frac{y}{1+y}\right)^{k} \sum_{\ell=0}^{\infty} \frac{(k m z)^{\ell}}{\ell !} \\
& =\frac{1}{1+y} e^{-a z} \sum_{k=0}^{\infty}\left(\frac{y}{1+y}\right)^{k} e^{k m z} \\
& =\frac{1}{1+y} \cdot \frac{e^{-a z}}{1-(y /(1+y)) e^{m z}} \\
& =\sum_{n=0}^{\infty} \widetilde{\mathscr{F}}_{m, a}(n ; m y) \frac{z^{n}}{n !} .
\end{aligned}
$$

Comparing the coefficients of $z^{n}$ yields (98). The proof is then completed when $m y$ is replaced with $x$ in (98).

Identity (98) used in the proof of the previous theorem is a generalization of Benoumhani's [3, Theorem 4]. On the other hand, when $x=1$,

$$
\widetilde{\mathscr{F}}_{m, a}(n)=\sum_{k=0}^{\infty} \frac{1}{2^{k+1}}(m k-a)^{n} .
$$

Moreover, we get the familiar representation of $\mathscr{F}_{m}(n ; 1)$ due to Rota [19] given by

$$
\widetilde{\mathscr{F}}_{m,-1}(n ; 1)=\sum_{k=0}^{\infty} \frac{1}{2^{k+1}}(m k+1)^{n}
$$

when $a=-1$ in (101).
It is well-known that the binomial coefficients $\left(\begin{array}{c}n \\ k\end{array}\right)$ satisfy the binomial inversion formula

$$
\begin{aligned}
& f_{k}=\sum_{j=0}^{k}\left(\begin{array}{l}
k \\
j
\end{array}\right) g_{j} \Longleftrightarrow \\
& g_{k}=\sum_{j=0}^{k}(-1)^{k-j}\left(\begin{array}{l}
k \\
j
\end{array}\right) f_{j} .
\end{aligned}
$$

The rest of this section contains corollaries which are obtained through the use of this.

Theorem 20. The noncentral Whitney numbers of the second kind satisfy the following recursion formula:

$$
\widetilde{W}_{m, a+1}(n, k)=\sum_{j=0}^{n}(-1)^{n-j}\left(\begin{array}{l}
n \\
j
\end{array}\right) \widetilde{W}_{m, a}(j, k) .
$$

Proof. Using the explicit formula (38) gives us

$$
\begin{aligned}
\widetilde{W}_{m, a+1}(n, k)=\frac{1}{m^{k} k !} \sum_{i=0}^{k}(-1)^{k-i}\left(\begin{array}{l}
k \\
i
\end{array}\right)(m i-a-1)^{n} \\
=\frac{1}{m^{k} k !} \sum_{i=0}^{k}(-1)^{k-i}\left(\begin{array}{l}
k \\
i
\end{array}\right) \sum_{j=0}^{n}\left(\begin{array}{l}
n \\
j
\end{array}\right)(m i-a)^{n-j}(-1)^{j} \\
=\sum_{j=0}^{n}(-1)^{j}\left(\begin{array}{l}
n \\
j
\end{array}\right) \\
\cdot \frac{1}{m^{k} k !} \sum_{i=0}^{k}\left(\begin{array}{l}
k \\
i
\end{array}\right)(-1)^{k-i}(m i-a)^{n-j} \\
=\sum_{j=0}^{n}(-1)^{j}\left(\begin{array}{l}
n \\
j
\end{array}\right) \widetilde{W}_{m, a}(n-j, k) .
\end{aligned}
$$

Reindexing the summation yields (104).

Applying the abovementioned binomial inversion formula for $g_{k}=\widetilde{W}_{m, a+1}(n, k)$ and $f_{j}=\widetilde{W}_{m, a}(j, k)$, we get the following corollary.

Corollary 21. The noncentral Whitney numbers of the second kind satisfy the relation given by

$$
\widetilde{W}_{m, a}(n, k)=\sum_{j=0}^{n}\left(\begin{array}{l}
n \\
j
\end{array}\right) \widetilde{W}_{m, a+1}(j, k) .
$$

Using (104), we have

$$
\begin{aligned}
& \sum_{k=0}^{n} \widetilde{W}_{m, a+1}(n, k) x^{k} \\
& =\sum_{k=0}^{n} \sum_{j=0}^{n}(-1)^{j}\left(\begin{array}{l}
n \\
j
\end{array}\right) \widetilde{W}_{m, a}(n-j, k) x^{k} \\
& =\sum_{j=0}^{n}(-1)^{n-j}\left(\begin{array}{c}
n \\
j
\end{array}\right) \sum_{k=0}^{n} \widetilde{W}_{m, a}(j, k) x^{k} .
\end{aligned}
$$


Similarly, we have

$$
\begin{aligned}
& \sum_{k=0}^{n} k ! \widetilde{W}_{m, a+1}(n, k) x^{k} \\
& =\sum_{k=0}^{n} \sum_{j=0}^{n}(-1)^{j}\left(\begin{array}{l}
n \\
j
\end{array}\right) k ! \widetilde{W}_{m, a}(n-j, k) x^{k} \\
& =\sum_{j=0}^{n}(-1)^{n-j}\left(\begin{array}{l}
n \\
j
\end{array}\right) \sum_{k=0}^{n} k ! \widetilde{W}_{m, a}(j, k) x^{k} .
\end{aligned}
$$

Thus, we have the following theorem.

Theorem 22. The noncentral Dowling and the noncentral Tanny-Dowling polynomials satisfy the following recursions:

$$
\begin{aligned}
& \widetilde{\mathscr{D}}_{m, a+1}(n ; x)=\sum_{j=0}^{n}(-1)^{n-j}\left(\begin{array}{l}
n \\
j
\end{array}\right) \widetilde{\mathscr{D}}_{m, a}(j ; x), \\
& \widetilde{\mathscr{F}}_{m, a+1}(n ; x)=\sum_{j=0}^{n}(-1)^{n-j}\left(\begin{array}{l}
n \\
j
\end{array}\right) \widetilde{\mathscr{F}}_{m, a}(j ; x) .
\end{aligned}
$$

Consequently, we have the following.

Corollary 23. The noncentral Dowling and Tanny-Dowling polynomials satisfy the relations given by

$$
\begin{aligned}
& \widetilde{\mathscr{D}}_{m, a}(n ; x)=\sum_{j=0}^{n}\left(\begin{array}{l}
n \\
j
\end{array}\right) \widetilde{\mathscr{D}}_{m, a+1}(j ; x), \\
& \widetilde{\mathscr{F}}_{m, a}(n ; x)=\sum_{j=0}^{n}\left(\begin{array}{l}
n \\
j
\end{array}\right) \widetilde{\mathscr{F}}_{m, a+1}(j ; x) .
\end{aligned}
$$

Theorem 24. For nonnegative real number $m$, the noncentral Whitney numbers of the second kind satisfy

$$
\begin{aligned}
& \widetilde{W}_{m+1, a}(n, k) \\
& =\frac{1}{(m+1)^{k} m^{n-k}} \sum_{j=0}^{n}\left(\begin{array}{c}
n \\
j
\end{array}\right) a^{n-j}(m+1)^{j} \widetilde{W}_{m, a}(j, k) .
\end{aligned}
$$

Proof. We can rewrite the explicit formula (38) as

$$
\begin{aligned}
& \widetilde{W}_{m+1, a}(n, k)=\frac{(m+1)^{n-k}}{k !} \\
& \cdot \sum_{i=0}^{k}(-1)^{k-i}\left(\begin{array}{l}
k \\
i
\end{array}\right)\left(i-\frac{a}{m+1}\right)^{n}=\frac{(m+1)^{n-k}}{k !} \\
& \cdot \sum_{i=0}^{k}(-1)^{k-i}\left(\begin{array}{l}
k \\
i
\end{array}\right)\left(i-\frac{a}{m}+\frac{a}{m(m+1)}\right)^{n} \\
& =\frac{(m+1)^{n-k}}{k !} \sum_{i=0}^{k}(-1)^{k-i}\left(\begin{array}{c}
k \\
i
\end{array}\right) \sum_{j=0}^{n}\left(\begin{array}{c}
n \\
j
\end{array}\right)\left(i-\frac{a}{m}\right)^{n-j}
\end{aligned}
$$

$$
\begin{aligned}
& \cdot\left(\frac{a}{m(m+1)}\right)^{j}=(m+1)^{n} \\
& \cdot \sum_{j=0}^{n}\left(\begin{array}{l}
n \\
j
\end{array}\right) \frac{m^{k}}{(m+1)^{k} m^{n-j}}\left(\frac{a}{m(m+1)}\right)^{j} \cdot \frac{1}{m^{k} k !} \\
& \cdot \sum_{i=0}^{k}\left(\begin{array}{l}
k \\
i
\end{array}\right)(-1)^{k-i}(m i-a)^{n-j}=\frac{1}{(m+1)^{k} m^{n-k}} \\
& \cdot \sum_{j=0}^{n}\left(\begin{array}{l}
n \\
j
\end{array}\right) a^{j}(m+1)^{n-j} \widetilde{W}_{m, a}(n-j, k) .
\end{aligned}
$$

Reindexing the summation yields (113).

The next corollary is easily obtained by applying binomial inversion formula to (113).

Corollary 25. The noncentral Whitney numbers of the second kind satisfy the relation given by

$$
\begin{aligned}
& \widetilde{W}_{m, a}(n, k) \\
& =\frac{1}{(m+1)^{n-k}} \sum_{j=0}^{n}\left(\begin{array}{l}
n \\
j
\end{array}\right)(-a)^{n-j}(m)^{j-k} \widetilde{W}_{m+1, a}(j, k) .
\end{aligned}
$$

Theorem 26. The noncentral Dowling and the noncentral Tanny-Dowling polynomials satisfy the following recursions:

$$
\begin{aligned}
& \widetilde{\mathscr{D}}_{m+1, a}(n ; x) \\
& =\frac{1}{m^{n}} \sum_{j=0}^{n} a^{n-j}\left(\begin{array}{l}
n \\
j
\end{array}\right)(m+1)^{j} \widetilde{\mathscr{D}}_{m, a}\left(j ; \frac{m}{m+1} x\right), \\
& \widetilde{\mathscr{F}}_{m+1, a}(n ; x) \\
& \quad=\frac{1}{m^{n}} \sum_{j=0}^{n} a^{n-j}\left(\begin{array}{l}
n \\
j
\end{array}\right)(m+1)^{j} \widetilde{\mathscr{F}}_{m, a}\left(j ; \frac{m}{m+1} x\right) .
\end{aligned}
$$

Proof. Combining (89) and (113) gives us

$$
\begin{aligned}
& \widetilde{\mathscr{D}}_{m+1, a}(n ; x)=\sum_{k=0}^{n} \widetilde{W}_{m+1, a}(n, k) x^{k} \\
& =\sum_{k=0}^{n} \frac{1}{(m+1)^{k} m^{n-k}} \\
& \cdot \sum_{j=0}^{n}\left(\begin{array}{c}
n \\
j
\end{array}\right) a^{j}(m+1)^{n-j} \widetilde{W}_{m, a}(n-j, k) x^{k}=\frac{1}{m^{n}} \\
& \cdot \sum_{j=0}^{n} a^{j}\left(\begin{array}{c}
n \\
j
\end{array}\right)(m+1)^{n-j} \sum_{k=0}^{n} \widetilde{W}_{m, a}(n-j, k) \\
& \cdot\left(\frac{m x}{m+1}\right)^{k}=\frac{1}{m^{n}} \sum_{j=0}^{n} a^{n-j}\left(\begin{array}{c}
n \\
j
\end{array}\right)(m+1)^{j}
\end{aligned}
$$




$$
\begin{aligned}
& \cdot \sum_{k=0}^{n} \widetilde{W}_{m, a}(j, k)\left(\frac{m x}{m+1}\right)^{k}=\frac{1}{m^{n}} \\
& \cdot \sum_{j=0}^{n} a^{n-j}\left(\begin{array}{l}
n \\
j
\end{array}\right)(m+1)^{j} \widetilde{\mathscr{D}}_{m, a}\left(j, \frac{m x}{m+1}\right) .
\end{aligned}
$$

Similarly, (90) and (113) give us

$$
\begin{aligned}
\widetilde{\mathscr{F}}_{m+1, a}(n ; x)=\sum_{k=0}^{n} k ! \widetilde{W}_{m+1, a}(n, k) x^{k} \\
=\sum_{k=0}^{n} \frac{k !}{(m+1)^{k} m^{n-k}} \sum_{j=0}^{n}\left(\begin{array}{c}
n \\
j
\end{array}\right) a^{j}(m+1)^{n-j} \widetilde{W}_{m, a} \\
\cdot(n-j, k) x^{k}=\frac{1}{m^{n}} \sum_{j=0}^{n} a^{j}\left(\begin{array}{l}
n \\
j
\end{array}\right)(m+1)^{n-j} \\
\cdot \sum_{k=0}^{n} k ! \widetilde{W}_{m, a}(n-j, k)\left(\frac{m x}{m+1}\right)^{k}=\frac{1}{m^{n}} \\
\cdot \sum_{j=0}^{n} a^{n-j}\left(\begin{array}{c}
n \\
j
\end{array}\right)(m+1)^{j} \sum_{k=0}^{n} k ! \widetilde{W}_{m, a}(j, k)\left(\frac{m x}{m+1}\right)^{k} \\
=\frac{1}{m^{n}} \sum_{j=0}^{n} a^{n-j}\left(\begin{array}{c}
n \\
j
\end{array}\right)(m+1)^{j} \widetilde{\mathscr{F}}_{m, a}\left(j, \frac{m x}{m+1}\right) .
\end{aligned}
$$

Remark 27. When $a=-r$, we get

$$
\begin{aligned}
& D_{m+1, r}(n ; x) \\
& =\frac{1}{m^{n}} \sum_{j=0}^{n}(-r)^{n-j}\left(\begin{array}{c}
n \\
j
\end{array}\right)(m+1)^{j} D_{m, r}\left(j ; \frac{m}{m+1} x\right), \\
& \widetilde{\mathscr{F}}_{m+1,-r}(n ; x) \\
& =\frac{1}{m^{n}} \sum_{j=0}^{n}(-r)^{n-j}\left(\begin{array}{c}
n \\
j
\end{array}\right)(m+1)^{j} \widetilde{\mathscr{F}}_{m,-r}\left(j ; \frac{m}{m+1} x\right),
\end{aligned}
$$

where $D_{m, r}(n ; x)$ are the $r$-Dowling polynomials [10, 17]. Moreover, the case where $a=-1$ yields [17, Theorems 2 and 3]

$$
\begin{aligned}
& D_{m+1}(n ; x) \\
& \quad=\frac{1}{m^{n}} \sum_{j=0}^{n}(-1)^{n-j}\left(\begin{array}{l}
n \\
j
\end{array}\right)(m+1)^{j} D_{m}\left(j ; \frac{m}{m+1} x\right), \\
& \mathscr{F}_{m+1}(n ; x) \\
& \quad=\frac{1}{m^{n}} \sum_{j=0}^{n}(-r)^{n-j}\left(\begin{array}{c}
n \\
j
\end{array}\right)(m+1)^{j} \mathscr{F}_{m}\left(j ; \frac{m}{m+1} x\right) .
\end{aligned}
$$
ing.
Corollary 28. The noncentral Dowling and Tanny-Dowling polynomials satisfy the relations given by

$$
\begin{aligned}
& \widetilde{\mathscr{D}}_{m, a}\left(n ; \frac{m}{m+1} x\right) \\
& =\frac{1}{(m+1)^{n}} \sum_{j=0}^{n}(-a)^{n-j}\left(\begin{array}{l}
n \\
j
\end{array}\right) m^{j} \widetilde{\mathscr{D}}_{m+1, a}(j ; x), \\
& \widetilde{\mathscr{F}}_{m, a}\left(n ; \frac{m}{m+1} x\right) \\
& =\frac{1}{(m+1)^{n}} \sum_{j=0}^{n}(-a)^{n-j}\left(\begin{array}{l}
n \\
j
\end{array}\right) m^{j} \widetilde{\mathscr{F}}_{m+1, a}(j ; x) .
\end{aligned}
$$

The next theorem and corollary can be obtained by similar method as the previous ones. The proof is left as exercise.

Theorem 29. The following recursion formulas hold:

$$
\begin{aligned}
& \widetilde{W}_{m+1, a+1}(n, k)=\frac{1}{(m+1)^{k} m^{n-k}} \sum_{j=0}^{n}\left(\begin{array}{l}
n \\
j
\end{array}\right)(a-m)^{n-j} \\
& \cdot(m+1)^{j} \widetilde{W}_{m, a}(j, k) \text {, } \\
& \widetilde{\mathscr{D}}_{m+1, a+1}(n ; x)=\frac{1}{m^{n}} \sum_{j=0}^{n}(a-m)^{n-j}\left(\begin{array}{l}
n \\
j
\end{array}\right)(m+1)^{j} \\
& \text { - } \widetilde{\mathscr{D}}_{m, a}\left(j ; \frac{m}{m+1} x\right) \text {, } \\
& \widetilde{\mathscr{F}}_{m+1, a+1}(n ; x)=\frac{1}{m^{n}} \sum_{j=0}^{n}(a-m)^{n-j}\left(\begin{array}{l}
n \\
j
\end{array}\right)(m+1)^{j} \\
& \cdot \widetilde{\mathscr{F}}_{m, a}\left(j ; \frac{m}{m+1} x\right) \text {. }
\end{aligned}
$$

Corollary 30. The following recursion formulas hold:

$$
\begin{aligned}
& \widetilde{W}_{m, a}(n, k)=\frac{1}{(m+1)^{n-k} m^{k}} \sum_{j=0}^{n}\left(\begin{array}{c}
n \\
j
\end{array}\right)(m-a)^{n-j} \\
& \cdot m^{j} \widetilde{W}_{m+1, a+1}(j, k) \text {, } \\
& \widetilde{\mathscr{D}}_{m, a}\left(n ; \frac{m}{m+1} x\right)=\frac{1}{(m+1)^{n}} \sum_{j=0}^{n}(m-a)^{n-j} \\
& \cdot\left(\begin{array}{l}
n \\
j
\end{array}\right) m^{j} \widetilde{\mathscr{D}}_{m+1, a+1}(j ; x) \text {, } \\
& \widetilde{\mathscr{F}}_{m, a}\left(n ; \frac{m}{m+1} x\right)=\frac{1}{(m+1)^{n}} \sum_{j=0}^{n}(m-a)^{n-j} \\
& \cdot\left(\begin{array}{l}
n \\
j
\end{array}\right) m^{j} \widetilde{\mathscr{F}}_{m+1, a+1}(j ; x) .
\end{aligned}
$$

The $n$th Bell polynomial

$$
\phi_{n}(x)=\sum_{k=0}^{n}\left\{\begin{array}{l}
n \\
k
\end{array}\right\} x^{k}
$$


is known to satisfy the explicit formula

$$
\phi_{n}(x)=\left(\frac{1}{e}\right)^{x} \sum_{i=0}^{\infty} \frac{i^{n}}{i !} x^{i} .
$$

Now, from (50), we have

$$
\begin{aligned}
\widetilde{\mathscr{D}}_{m, a}(n ; x) & =\sum_{k=0}^{n} \sum_{j=0}^{n}\left(\begin{array}{l}
n \\
j
\end{array}\right)(-a)^{n-j} m^{j-k}\left\{\begin{array}{l}
j \\
k
\end{array}\right\} x^{k} \\
& =\sum_{j=0}^{n}\left(\begin{array}{l}
n \\
j
\end{array}\right)(-a)^{n-j} m^{j} \sum_{k=0}^{n}\left\{\begin{array}{l}
j \\
k
\end{array}\right\}\left(\frac{x}{m}\right)^{k} .
\end{aligned}
$$

Thus we have the next theorem.

Theorem 31. The noncentral Dowling polynomials satisfy

$$
\widetilde{\mathscr{D}}_{m, a}(n ; x)=\sum_{j=0}^{n}(-a)^{n-j}\left(\begin{array}{l}
n \\
j
\end{array}\right) m^{j} \phi_{j}\left(\frac{x}{m}\right) .
$$

Corollary 32. The Bell polynomials satisfy the following identity:

$$
\phi_{n}\left(\frac{x}{m}\right)=\frac{1}{m^{n}} \sum_{j=0}^{n} a^{n-j}\left(\begin{array}{l}
n \\
j
\end{array}\right) \widetilde{\mathscr{D}}_{m, a}(j ; x) .
$$

The case where $a=-1$ in (127) and (128) is due to Rahmani (cf. [17, Theorem 4 and Corollary 1]). Obviously, $\widetilde{\mathscr{D}}_{m, a}(n ; x)$ is the binomial transform of $a^{n-j} m^{j} \phi_{j}(x / m)$. The curious identity

$$
\begin{aligned}
& \sum_{k=0}^{\ell}\left(\begin{array}{l}
\ell \\
k
\end{array}\right)\left(\begin{array}{c}
n+k \\
s
\end{array}\right) \alpha_{n+k-s} \\
& \quad=\sum_{k=0}^{\ell}\left(\begin{array}{l}
n \\
k
\end{array}\right)\left(\begin{array}{c}
\ell+k \\
s
\end{array}\right)(-1)^{n-k} \beta_{\ell+k-s}
\end{aligned}
$$

is due to Chen [20, Theorem 3.2]. When $\ell=s=n$, and if we let $\alpha_{k}=a^{n-k} m^{k} \phi_{k}(x / m)$ and $\beta_{k}=\widetilde{\mathscr{D}}_{m, a}(k ; x)$, then we have the following.

Corollary 33. The noncentral Dowling polynomials and the Bell polynomials can be related as follows:

$$
\begin{aligned}
\sum_{k=0}^{n}\left(\begin{array}{c}
n \\
k
\end{array}\right)\left(\begin{array}{c}
n+k \\
s
\end{array}\right) a^{n-k} m^{k} \phi_{k}\left(\frac{x}{m}\right) \\
=\sum_{k=0}^{n}\left(\begin{array}{l}
n \\
k
\end{array}\right)\left(\begin{array}{c}
n+k \\
s
\end{array}\right)(-1)^{n-k} \widetilde{\mathscr{D}}_{m, a}(k ; x) .
\end{aligned}
$$

\section{The Hankel Transform of Noncentral Dowling Numbers}

Hankel matrices had been studied by several mathematicians because of their connections in some areas of mathematics, physics, and computer science. Further theories and applications of these matrices have been established including the Hankel determinant and Hankel transform. The Hankel transform was first introduced in Sloane's sequence A055878 [21] and was later on studied by Layman [22]. Layman [22] first defined the Hankel transform of an integer sequence as the sequence of Hankel determinants of order $n$ of a given sequence. Among the remarkable properties established by Layman [22] is the property that any integer sequence has the same Hankel transform as its binomial transform, as well as its invert transform. In this section, we thoroughly investigate the Hankel transform of the noncentral Dowling numbers using this property.

Let $\Gamma=\left(b_{n, k}\right)$ be the infinite lower triangular matrix defined recursively by

$$
b_{n, k}=b_{n-k, k-1}+(m k+1) b_{n-1, k}+m(k+1) b_{n-1, k+1},
$$

where $n \geq 1, b_{0,0}=1, b_{0, k}=0$ if $k>0$ and $b_{n, k}=0$ if $n<k$.

The next proposition shows that (131) is a recurrence relation of the infinite lower triangular matrix $\Gamma=\left(b_{n, k}\right)$, where the entries in the 0 -column are the numbers $\widetilde{D}_{m, 0}(n)$.

Proposition 34. Let $\Phi_{k}(z)$ be the exponential generating function of the kth column of matrix $\Gamma$. Then

$$
\Phi_{k}(z)=e^{m^{-1}\left(e^{m z}-1\right)} \frac{\left(e^{m z}-1\right)^{k}}{m^{k} k !},
$$

where $k \geq 0$ and the 0 -column entries of $\Gamma$ are the numbers $\widetilde{D}_{m, 0}(n)$.

To obtain the Hankel transform of the noncentral Dowling numbers, the next lemma which may be proved by induction is essential.

Lemma 35. Let $c_{n}$ be the $n$th row of $\Gamma=\left(b_{n, k}\right)$. Define

$$
c_{n} \circ c_{p}=\sum_{k \geq 0} b_{n, k} b_{p, k} m^{k} k !
$$

Then for all nonnegative integers $n$ and $p$

$$
c_{n} \circ c_{p}=b_{n+p, 0}=\widetilde{D}_{m, 0}(n+p) .
$$

Before stating the next theorem, we first let

$$
\begin{aligned}
& \mathfrak{D}_{m, a} \\
& \quad=\left(\begin{array}{cccc}
\widetilde{\mathscr{D}}_{m, a}(0) & \widetilde{\mathscr{D}}_{m, a}(1) & \ldots & \widetilde{\mathscr{D}}_{m, a}(n) \\
\widetilde{\mathscr{D}}_{m, a}(1) & \widetilde{\mathscr{D}}_{m, a}(2) & \cdots & \widetilde{\mathscr{D}}_{m, a}(n+1) \\
\vdots & \vdots & \cdots & \vdots \\
\widetilde{\mathscr{D}}_{m, a}(n) & \widetilde{\mathscr{D}}_{m, a}(n+1) & \cdots & \widetilde{\mathscr{D}}_{m, a}(2 n)
\end{array}\right)
\end{aligned}
$$

and $H\left\{\mathfrak{D}_{m, a}\right\}$ be the Hankel transform of the numbers $\widetilde{\mathscr{D}}_{m, a}(n)$.

Theorem 36. The Hankel transform of the numbers $\widetilde{\mathscr{D}}_{m, 0}(n)$ is given by

$$
H\left\{\mathfrak{D}_{m, 0}\right\}=m^{\left(\begin{array}{c}
n+1 \\
2
\end{array}\right)} \prod_{j \geq 0} j ! .
$$


Proof. Suppose $\Gamma_{n}$ is the lower triangular submatrix of $\Gamma$ consisting of the rows and columns numbered from 0 to $n$. Then $\operatorname{det} \Gamma_{n}^{T}=1$. Let $\bar{\Gamma}_{n}=\left(m^{j} j ! b_{i, j}\right)_{0 \leq i, i \leq n}$. It implies that

$$
\operatorname{det} \bar{\Gamma}_{n}=\prod_{j=0}^{n} m^{j} j !
$$

On the other hand, by (134), we have $\bar{\Gamma}_{n} \cdot \Gamma_{n}^{T}=\left(c_{i, j}\right)_{0 \leq i, j \leq n}$, where

$$
c_{i, j}=\sum_{k \geq 0} b_{i, j} b_{j, k} m^{j} j !=b_{i+j, 0}=\widetilde{D}_{m, 0}(i+j) .
$$

That is, $\bar{\Gamma}_{n} \cdot \Gamma_{n}^{T}=\left(\widetilde{D}_{m, 0}(i+j)\right)_{0 \leq i, j \leq n}$. Thus, we have

$$
\begin{aligned}
\operatorname{det}\left(\bar{\Gamma}_{n} \cdot \Gamma_{n}^{T}\right) & =\left(\operatorname{det} \bar{\Gamma}_{n}\right)\left(\operatorname{det} \Gamma_{n}^{T}\right)=\prod_{j=0}^{n} m^{j} j ! \\
& =m^{n(n+1) / 2} \prod_{j=0}^{n} j !=m^{\left(\begin{array}{c}
n+1 \\
2
\end{array}\right)} \prod_{j=0}^{n} j ! .
\end{aligned}
$$

This is the desired result.

Notice that by (109) and (111), $\widetilde{D}_{m, a+1}(n)$ is the binomial transform of $\widetilde{D}_{m, a}(n)$. Hence by the abovementioned property of Layman's [22], $\widetilde{D}_{m, 0}(n)$ and $\widetilde{D}_{m, a}(n)$ have the same Hankel transform. Finally, we have the following theorem.

Theorem 37. The Hankel transform of the noncentral Dowling numbers is given by

$$
H\left\{\mathfrak{D}_{m, a}\right\}=m^{\left(\begin{array}{c}
n+1 \\
2
\end{array}\right)} \prod_{j \geq 0} j ! .
$$

\section{More Theorems on $\widetilde{\mathscr{D}}_{m, a}(n ; x)$}

Proposition 38. The noncentral Whitney numbers of the second kind satisfy the rational generating function

$$
\sum_{n=k}^{\infty} \widetilde{W}_{m, a}(n, k) z^{n-k}=\frac{1}{\prod_{i=0}^{k}(1-(m i-a) z)} .
$$

It is easy to express this identity as

$$
\begin{aligned}
\sum_{n=k}^{\infty} \widetilde{W}_{m, a}(n, k) z^{n}= & \frac{1}{m^{k}(1+a z)} \\
& \cdot \frac{(-1)^{k}}{\langle((m-a) z-1) / m z\rangle_{k}},
\end{aligned}
$$

where $\langle x\rangle_{k}=x(x+1)(x+2) \cdots(x+k-1)$. Hence, we have

$$
\begin{aligned}
\sum_{k=0}^{\infty} & \left(\sum_{n=k}^{\infty} \widetilde{W}_{m, a}(n, k) z^{n}\right) x^{k} \\
& =\frac{1}{1+a z} \sum_{k=0}^{\infty} \frac{\langle 1\rangle_{k}}{\langle((m-a) z-1) / m z\rangle_{k}} \cdot \frac{(-x / m)^{k}}{k !} .
\end{aligned}
$$

Since the left-hand side is just

$$
\sum_{n=0}^{\infty}\left(\sum_{k=0}^{\infty} \widetilde{W}_{m, a}(n, k) x^{k}\right) z^{n}=\sum_{n=0}^{\infty} \widetilde{\mathscr{D}}_{m, a}(n ; x) z^{n}
$$

then by using the hypergeometric function defined by

$$
\begin{aligned}
{ }_{p} F_{q} & \left(\begin{array}{c}
a_{1}, a_{2}, \ldots, a_{p} \\
b_{1}, b_{2}, \ldots, b_{q}
\end{array}\right) \\
= & \sum_{k=0}^{\infty} \frac{\left\langle a_{1}\right\rangle_{k}\left\langle a_{2}\right\rangle_{k} \cdots\left\langle a_{p}\right\rangle_{k}}{\left\langle b_{1}\right\rangle_{k}\left\langle b_{2}\right\rangle_{k} \cdots\left\langle b_{q}\right\rangle_{k}} \frac{t^{k}}{k !},
\end{aligned}
$$

we get

$$
\begin{aligned}
& \sum_{n=0}^{\infty} \widetilde{\mathscr{D}}_{m, a}(n ; x) z^{n} \\
& \quad=\frac{1}{1+a z}{ }_{1} F_{1}\left(\frac{(m-a) z-1}{m z} \mid-\frac{x}{m}\right) .
\end{aligned}
$$

Theorem 39. The noncentral Dowling polynomials satisfy the generating function

$$
\begin{aligned}
& \sum_{n=0}^{\infty} \widetilde{\mathscr{D}}_{m, a}(n ; x) z^{n} \\
& \quad=\frac{1}{1+a z}\left(\frac{1}{e}\right)^{x / m}{ }_{1} F_{1}\left(\frac{\frac{-a z-1}{m z}}{\frac{(m-a) z-1}{m z}} \mid \frac{x}{m}\right) .
\end{aligned}
$$

Proof. Applying Kummer's formula [23, page 505] given by

$$
e^{-x}{ }_{1} F_{1}\left(\begin{array}{l}
\alpha \\
\beta
\end{array}\right)={ }_{1} F_{1}\left(\begin{array}{cc}
\beta-\alpha & \\
\beta & \mid-x
\end{array}\right)
$$

to (146) with $\alpha=(-a z-1) / m z$ and $\beta=((m-a) z-1) / m z$ yields the desired result.

Remark 40. When $m=1$ and $a=-r$, we get [24, Theorem 3.2]

$$
\begin{aligned}
& \frac{-1}{r z-1}\left(\frac{1}{e}\right)^{x}{ }_{1} F_{1}\left(\frac{\frac{r z-1}{z}}{\frac{r z+z-1}{m z}} \mid \frac{x}{m}\right) \\
& =\sum_{n=0}^{\infty} \phi_{n, r}(x) z^{n},
\end{aligned}
$$

where $\phi_{n, r}(x) z^{n}$ denotes the $r$-Bell polynomials. In a similar manner, we get [7, page 10$]$

$$
\left(\frac{1}{e}\right)^{x / \alpha}{ }_{1} F_{1}\left(\begin{array}{c|c}
-\frac{1}{\alpha z} & \frac{x}{\alpha z-1} \\
\frac{\alpha z}{\alpha}
\end{array}\right)=\sum_{n=0}^{\infty} \widetilde{D}_{(\alpha)}(n ; x) z^{n}
$$


where $\widetilde{D}_{(\alpha)}(n ; x)$ denotes the translated Dowling polynomials if we set $m=\alpha$ and $a=0$.

An equivalent of the result in the previous theorem is due to R. B. Corcino and C. B. Corcino [25, Theorem 4.1] obtained when $m=\beta$ and $a=-r$. This identity is established for the $(r, \beta)$-Bell polynomials $G_{n, \beta, r}(x)$.

Definition 41 (see [26, page 268]). A real sequence $v_{k}, k=$ $0,1,2, \ldots$ is called convex on an interval $[a, b]$, where $[a, b]$ contains at least 3 consecutive integers, if

$$
v_{k} \leq \frac{1}{2}\left(v_{k-1}+v_{k+1}\right), \quad k \in[a+1, b-1] .
$$

The above inequality is often referred to as the convexity property.

Theorem 42. For $a, m \geq 0$, the sequence of noncentral Dowling polynomials $\widetilde{D}_{m, a}(n ; x), x>0$, satisfies the convexity property.

Proof. Suppose $m k-a \geq 0$. Then

$$
\begin{aligned}
0 & \leq[1-(m k-a)]^{2}, \\
0 & \leq 1-2(m k-a)+(m k-a)^{2}, \\
m k-a & \leq \frac{1}{2}\left[1+(m k-a)^{2}\right], \\
(m k-a)^{n+1} & \leq \frac{1}{2}\left[(m k-a)^{n}+(m k-a)^{n+2}\right] .
\end{aligned}
$$

Multiplying the above inequality by $(x / m)^{i}(1 / i !)$ and summing over $i$ give

$$
\widetilde{\mathscr{D}}_{m, a}(n+1 ; x) \leq \frac{1}{2}\left[\widetilde{\mathscr{D}}_{m, a}(n ; x)+\widetilde{\mathscr{D}}_{m, a}(n+2 ; x)\right] .
$$

This is precisely the desired result.

Cesàro [27] obtained an integral representation of the Bell numbers $\phi_{n}:=\phi_{n}(1)$, namely,

$$
\phi_{n}=\frac{2 n !}{\pi e} \operatorname{Im} \int_{0}^{\pi} e^{e^{i \theta}} \sin (n \theta) d \theta .
$$

Several generalizations of this remarkable representation were presented by Mező [24], Mangontarum et al. [6], and R. B. Corcino and C. B. Corcino [25]. To establish an analogous representation for $\widetilde{\mathscr{D}}_{m, a}(n ; x)$, we take the explicit formula in (38) and substitute it to the right-hand side of Callan's [28] integral identity given by

$$
\operatorname{Im} \int_{0}^{\pi} e^{j e^{i \theta}} \sin (n \theta) d \theta=\frac{\pi}{2} \frac{j^{n}}{n !} .
$$

That is, we obtain

$$
\begin{gathered}
\widetilde{W}_{m, a}(n, k)=\frac{2 n !}{m^{k} k ! \pi} \sum_{j=0}^{k}(-1)^{k-j}\left(\begin{array}{l}
k \\
j
\end{array}\right) \operatorname{Im} \int_{0}^{\pi} e^{(m j-a) e^{i \theta}} \\
\cdot \sin (n \theta) d \theta=\frac{2 n !}{m^{k} k ! \pi} \\
\cdot \operatorname{Im} \int_{0}^{\pi}\left[\sum_{j=0}^{k}(-1)^{k-j}\left(\begin{array}{l}
k \\
j
\end{array}\right)\left(e^{m e^{i \theta}}\right)^{j}\right] e^{-a e^{i \theta}} \\
\cdot \sin (n \theta) d \theta=\frac{2 n !}{\pi} \\
\cdot \operatorname{Im} \int_{0}^{\pi} \frac{\left(\left(e^{m e^{i \theta}}-1\right) / m\right)^{k}}{k !} e^{-a e^{i \theta}} \sin (n \theta) d \theta .
\end{gathered}
$$

Multiplying both sides by $x^{k}$ and summing over $k$ give

$$
\begin{aligned}
& \sum_{k=0}^{\infty} \widetilde{W}_{m, a}(n, k) x^{k}=\frac{2 n !}{\pi} \\
& \cdot \operatorname{Im} \int_{0}^{\pi}\left\{\sum_{k=0}^{\infty} \frac{\left(\left(\left(e^{m e^{i \theta}}-1\right) / m\right) x\right)^{k}}{k !}\right\} e^{-a e^{i \theta}} \\
& \cdot \sin (n \theta) d \theta=\frac{2 n !}{\pi} \operatorname{Im} \int_{0}^{\pi} e^{\left(e^{m e^{i \theta}}-1\right) x / m-a e^{i \theta}} \\
& \cdot \sin (n \theta) d \theta .
\end{aligned}
$$

Hence,

$$
\widetilde{D}_{m, a}(n ; x)=\frac{2 n !}{\pi e^{x / m}} \operatorname{Im} \int_{0}^{\pi} e^{x\left(e^{m e^{i \theta}} / m\right)-a e^{i \theta}} \sin (n \theta) d \theta
$$

and we have the following theorem.

Theorem 43. The noncentral Dowling polynomials have the integral representation

$$
\begin{aligned}
& \widetilde{\mathscr{D}}_{m, a}(n ; x) \\
& \quad=\frac{2 n !}{\pi e^{x / m}} \operatorname{Im} \int_{0}^{\pi} e^{x m^{-1} e^{m e^{i \theta}}} e^{-a e^{i \theta}} \sin (n \theta) d \theta .
\end{aligned}
$$

Remark 44. The earlier mentioned results due to Mezö, Mangontarum et al., and R. B. Corcino and C. B. Corcino can be obtained from this theorem by carefully assigning values to the parameters $m, a$, and $x$ (cf. [24, Theorem 6.1], [6, Theorems 10], and [25, Equation (4.12)]).

Another interesting fact on the Bell polynomials is the relation

$$
E_{\lambda}\left[X^{n}\right]=\phi_{n}(\lambda),
$$


where $E_{\lambda}\left[X^{n}\right]$ denotes the $n$th moment of a Poisson random variable $X$ with mean $\lambda$. From this point on, we use $X$ to denote such random variable. Another identity in line with this was obtained by Privault [29], namely,

$$
E_{\lambda}\left[(X+y-\lambda)^{n}\right]=\phi_{n}(y,-\lambda),
$$

where $\phi_{n}(y, \lambda)$ is an extension of Bell polynomials satisfying

$$
\begin{aligned}
\sum_{k=0}^{\infty} \phi_{n}(y, \lambda) \frac{t^{k}}{k !} & =e^{t y-\lambda\left(e^{t}-t-1\right)}, \\
\phi_{n}(y,-\lambda) & =\sum_{k=0}^{n}\left(\begin{array}{l}
n \\
k
\end{array}\right)(y-\lambda)^{n-k} \sum_{j=0}^{k}\left\{\begin{array}{l}
k \\
j
\end{array}\right\} \lambda^{j} .
\end{aligned}
$$

It is also known that the $n$th factorial moment of $X$ is

$$
E_{\lambda}\left[(X)_{n}\right]=\lambda^{n}
$$

Now, if we take the expectation of (40), we get

$$
\begin{gathered}
E_{\lambda}\left[(m X-a)^{n}\right]=\sum_{k=0}^{\infty} m^{k} \widetilde{W}_{m, a}(n, k) E_{\lambda}\left[(X)_{k}\right] \\
=\sum_{k=0}^{\infty} \frac{1}{k !} \sum_{j=0}^{k}(-1)^{j}\left(\begin{array}{l}
k \\
j
\end{array}\right)(m(j-k)-a)^{n} \lambda^{k} \\
=\sum_{j=0}^{\infty} \sum_{k=j}^{\infty} \frac{(-1)^{j}(m(j-k)-a)^{n} \lambda^{k}}{j !(k-j) !}
\end{gathered}
$$

through the aid of (38). Reindexing the sum yields

$$
\begin{aligned}
E_{\lambda}\left[(m X-a)^{n}\right] & =\sum_{j=0}^{\infty} \frac{(-1)^{j}}{j !} \sum_{i=0}^{\infty} \frac{(m i-a)^{n}}{i !} \lambda^{i} \\
& =e^{-\lambda} \sum_{i=0}^{\infty} \frac{(m i-a)^{n}}{i !} \lambda^{i} .
\end{aligned}
$$

Clearly, when $\lambda$ is replaced with $\lambda / m$,

$$
E_{\lambda / m}\left[(m X-a)^{n}\right]=\widetilde{\mathscr{D}}_{m, a}(n ; \lambda)
$$

On the other hand, using the binomial theorem,

$$
\begin{aligned}
E_{\lambda}\left[(m X-a)^{n}\right] & =\sum_{k=0}^{n}\left(\begin{array}{l}
n \\
k
\end{array}\right)(-a)^{n-k} m^{k} E_{\lambda}\left[X^{k}\right] \\
& =\sum_{k=0}^{n}\left(\begin{array}{l}
n \\
k
\end{array}\right)(-a)^{n-k} m^{k} \phi_{k}(\lambda) .
\end{aligned}
$$

The above results are compiled in the next theorem.

Theorem 45. The following identities hold:

$$
E_{\lambda}\left[(m X-a)^{n}\right]=e^{-\lambda} \sum_{i=0}^{\infty} \frac{(m i-a)^{n}}{i !} \lambda^{i},
$$

$$
\begin{aligned}
E_{\lambda}\left[(m X-a)^{n}\right] & =\sum_{k=0}^{n}\left(\begin{array}{l}
n \\
k
\end{array}\right)(-a)^{n-k} m^{k} \phi_{k}(\lambda), \\
E_{\lambda / m}\left[(m X-a)^{n}\right] & =\widetilde{\mathscr{D}}_{m, a}(n ; \lambda) .
\end{aligned}
$$

Remark 46. When $m=1$ and $a=0$ in (169), we get the classical identity (160). Similarly, if $m=1$ and $a=\lambda-y$ in (170), we recover Privault's identities in (161) and (163). Some results reported by Mangontarum and Corcino [30, Remarks 1, 2, and 3] can also be obtained from this theorem.

\section{Some Questions and Conjectures}

There are a number of further applications and possible extensions of the numbers introduced in this paper. The authors would like to direct the attention of the readers to some questions and conjectures.

Several studies regarding the identification of the index for which certain Stirling-type numbers attain their maximum value were conducted earlier by some mathematicians, for instance, Mező $[24,31]$ for the $r$-Stirling and $r$-Bell numbers, R. B. Corcino and C. B. Corcino [32] for the generalized Stirling numbers, and recently, Corcino et al. [33] for the noncentral Stirling numbers of the first kind.

Question 1. Is it possible to identify the maximizing index of the noncentral Whitney numbers of both kinds? Will these be different from the said earlier results?

Perhaps this question may be answered by the so-called "Erdo"s and Stone Theorem" mentioned in [33].

The study of asymptotic estimates/approximations and asymptotic formulas for Stirling-type numbers (such as the $(r, \beta)$-Stirling numbers and the $r$-Whitney numbers of the second kind) has been the interest of several mathematicians, especially C. B. Corcino and R. B. Corcino [34, 35], Corcino et al. [36], and Corcino et al. [37]. The next question is an interesting motivation for further study.

Question 2. It is compelling to study asymptotic approximations and obtain formulas for the noncentral Whitney numbers. However, will these formulas be distinct from those results found in [34-37] or will they be equivalent?

Corcino et al. [38] defined distinct "q-analogues" for the noncentral Stirling numbers of the second kind. Using Definition 47, the said $q$-noncentral Stirling numbers of the second kind were given a combinatorial interpretation in the context of $A$-tableaux. A more general study can actually be seen in the work of de Médicis and Leroux [39].

Definition 47 (see [39]). An $A$-tableau is a list $\Phi$ of columns $c$ of Ferrer's diagram of a partition $\Lambda$ (by decreasing order of length) such that the lengths $|c|$ are part of the sequence $A=$ $\left(a_{i}\right)_{i \geq 0}$, a strictly increasing sequence of nonnegative integers.

In line with this, denoting by $T^{A}(x, y)$ the set of $A$ tableaux with $A=\{0,1,2, \ldots, x\}$ and exactly $y$ columns and 
letting $\omega_{A}(\Phi)=\prod_{c \in \Phi} \omega(|c|), \Phi \in T^{A}(x, y)$, we have the following conjecture.

Conjecture 48. For complex numbers $m$ and $a$, and $\omega: N \rightarrow$ $K$ a function from the set of nonnegative integers $N$ to a ring $K$, defined by

$$
\omega(|c|)=m|c|-a
$$

where $|c|$ is the length of column cof an A-tableaux in $T^{A}(k, n-$ $k)$, one has

$$
\widetilde{W}_{m, a}(n, k)=\sum_{\Phi \in T^{A}(k, n-k)} \prod_{c \in \Phi} \omega(|c|) .
$$

The key to proving this remarkable observation might be achieved using the explicit formula in (52).

A "multiparameter version" of the noncentral Stirling numbers of Koutras [12] was introduced by El-Desouky [40]. This extended the number of parameters from the usual one that is $a$ to a sequence $a_{i}, i=0,1, \ldots, a_{n-1} \cdot q$ analogues of these numbers were then defined by Corcino and Mangontarum [41].

Conjecture 49. It is possible to establish a multiparameter version of the noncentral Whitney numbers of both kinds (may be called multiparameter noncentral Whitney numbers) either by means of a triangular recurrence relation or by a certain generating function.

Before ending this section, let us first note that Mangontarum and Katriel [42] investigated a connection of the defining relations of the $r$-Whitney numbers of both kinds in (9) and (10) and the Boson operators $a$ and $a^{\dagger}$ known to satisfy the commutation relation

$$
\left[a, a^{\dagger}\right] \equiv a a^{\dagger}-a^{\dagger} a=1 .
$$

Using their observations in this matter, they were able to define a remarkable $q$-deformation of the $r$-Whitney numbers using the $q$-Boson operators of Arik and Coon [43] which satisfies

$$
\left[a, a^{\dagger}\right]_{q} \equiv a a^{\dagger}-q a^{\dagger} a=1
$$

\section{Competing Interests}

The authors declare that no competing interests exist regarding the publication of this paper.

\section{Acknowledgments}

This study is supported by the Office of the Vice Chancellor for Academic Affairs and the Office of the Vice Chancellor for Research and Extension of Mindanao State University, Main Campus, Marawi City, the Office of the Campus Head of Mindanao State University, Maigo School of Arts and Trades, and the Office of the President, Mindanao State University, Main Campus, Marawi City.

\section{References}

[1] T. A. Dowling, "A class of geometric lattices based on finite groups," Journal of Combinatorial Theory. Series B, vol. 15, pp. 61-86, 1973.

[2] M. Benoumhani, "On Whitney numbers of Dowling lattices," Discrete Mathematics, vol. 159, no. 1-3, pp. 13-33, 1996.

[3] M. Benoumhani, "On some numbers related to Whitney numbers of Dowling lattices," Advances in Applied Mathematics, vol. 19, no. 1, pp. 106-116, 1997.

[4] M. Benoumhani, "Log-concavity of Whitney numbers of Dowling lattices," Advances in Applied Mathematics, vol. 22, no. 2, pp. $186-189,1999$.

[5] H. Belbachir and I. E. Bousbaa, "Translated Whitney and $r$ Whitney numbers: a combinatorial approach," Journal of Integer Sequences, vol. 16, article 13.8.6, 2013.

[6] M. M. Mangontarum, A. P. Macodi-Ringia, and N. S. Abdulcarim, "The translated Dowling polynomials and numbers," International Scholarly Research Notices, vol. 2014, Article ID 678408, 8 pages, 2014.

[7] M. M. Mangontarum and A. Dibagulun, "On the translated Whitney numbers and their combinatorial properties," British Journal of Applied Science and Technology, vol. 11, no. 5, pp. 1-15, 2015.

[8] D. Callan, Cesàro's Integral Formula for the Bell Numbers (Corrected), 2005, http://www.stat.wisc.edu/ callan/notes/ cesaro/cesaro.pdf.

[9] I. Mező, "A new formula for the Bernoulli polynomials," Results in Mathematics, vol. 58, no. 3-4, pp. 329-335, 2010.

[10] G.-S. Cheon and J.-H. Jung, "r-Whitney numbers of Dowling lattices," Discrete Mathematics, vol. 312, no. 15, pp. 2337-2348, 2012.

[11] I. Mező and J. L. Ramirez, "The linear algebra of the $r$-Whitney matices," Integral Transforms and Special Functions, vol. 26, no. 3, pp. 213-225, 2015.

[12] M. Koutras, "Non-central Stirling numbers and some applications," Discrete Mathematics, vol. 42, no. 1, pp. 73-89, 1982.

[13] A. Z. Broder, “The $r$-Stirling numbers," Discrete Mathematics, vol. 49, no. 3, pp. 241-259, 1984.

[14] R. L. Graham, D. E. Knuth, and O. Patashnik, Concrete Mathematics, Addison-Wesley, Reading, Mass, USA, 1989.

[15] J. Pan, "Matrix decomposition of the unified generalized Stirling numbers and inversion of the generalized factorial matrices," Journal of Integer Sequences, vol. 15, article 12.6.6, 2012.

[16] L. C. Hsu and P. J. Shiue, "A unified approach to generalized Stirling numbers," Advances in Applied Mathematics, vol. 20, no. 3, pp. 366-384, 1998.

[17] M. Rahmani, "Some results on Whitney numbers of Dowling lattices," Arab Journal of Mathematical Sciences, vol. 20, no. 1, pp. 11-27, 2014.

[18] S. Tanny, "On some numbers related to the Bell numbers," Canadian Mathematical Bulletin, vol. 17, pp. 733-738, 1975.

[19] G.-C. Rota, “The number of partitions of a set," The American Mathematical Monthly, vol. 71, pp. 498-504, 1964.

[20] K.-W. Chen, "Identities from the binomial transform," Journal of Number Theory, vol. 124, no. 1, pp. 142-150, 2007.

[21] N. J. Sloane, "Least positive sequence with Hankel transform $\{1,1,1,1,1, \ldots\}$," The On-Line Encyclopedia of Integer Sequences, July 2000, http://oeis.org. 
[22] J. W. Layman, "The Hankel transform and some of its properties," Journal of Integer Sequences, vol. 4, no. 1, Article ID 01.1.5, 2001.

[23] M. Abramowitz and I. A. Stegun, Eds., Handbook of Mathematical Functions with Formulas, Graphs, and Mathematical Tables, Dover, New York, NY, USA, 9th edition, 1972.

[24] I. Mező, “The $r$-Bell numbers," Journal of Integer Sequences, vol. 14, no. 1, Article ID 11.1.1, 2011.

[25] R. B. Corcino and C. B. Corcino, "On generalized Bell polynomials," Discrete Dynamics in Nature and Society, vol. 2011, Article ID 623456, 21 pages, 2011.

[26] L. Comtet, Advanced Combinatorics, D. Reidel, Dordrecht, Netherlands, 1974.

[27] M. Cesàro, "Sur une équation aux différences melées," Nouvelles Annales de Mathématiques, vol. 4, pp. 36-40, 1883.

[28] J. Stirling, Methodus Differentialissme Tractus de Summatione et Interpolatione Serierum Infinitarum, London, UK, 1730, (English translation by F. Holliday with the title: The Differential Method, London, UK, 1749).

[29] N. Privault, "Generalized Bell polynomials and the combinatorics of Poisson central moments," Electronic Journal of Combinatorics, vol. 18, no. 1, pp. 1-10, 2011.

[30] M. M. Mangontarum and R. B. Corcino, "The generalized factorial moments in terms of a Poisson random variable," GSTF Journal of Mathematics, Statistics and Operations Research, vol. 2, no. 1, pp. 64-67, 2013.

[31] I. Mezö, "On the maximum of $r$-Stirling numbers," Advances in Applied Mathematics, vol. 41, no. 3, pp. 293-306, 2008.

[32] R. B. Corcino and C. B. Corcino, "On the maximum of generalized Stirling numbers," Utilitas Mathematica, vol. 86, pp. 241-256, 2011.

[33] R. B. Corcino, C. B. Corcino, and P. B. Aranas, "The peak of noncentral Stirling numbers of the first kind," International Journal of Mathematics and Mathematical Sciences, vol. 2015, Article ID 982812, 7 pages, 2015.

[34] C. B. Corcino and R. B. Corcino, "Asymptotic estimates for second kind generalized stirling numbers," Journal of Applied Mathematics, vol. 2013, Article ID 918513, 7 pages, 2013.

[35] C. B. Corcino and R. B. Corcino, "An asymptotic formula for rBell numbers with real arguments," ISRN Discrete Mathematics, vol. 2013, Article ID 274697, 7 pages, 2013.

[36] C. B. Corcino, R. B. Corcino, and N. Acala, "Asymptotic estimates for r-Whitney numbers of the second kind," Journal of Applied Mathematics, vol. 2014, Article ID 354053, 7 pages, 2014.

[37] C. B. Corcino, R. B. Corcino, and R. J. Gasparin, "Equivalent asymptotic formulas of second kind r-Whitney numbers," Integral Transforms and Special Functions, vol. 26, no. 3, pp. 192-202, 2015.

[38] C. B. Corcino, R. B. Corcino, J. M. Ontolan, C. M. PerezFernandez, and E. R. Cantallopez, "The Hankel transform of $q$ noncentral Bell numbers," International Journal of Mathematics and Mathematical Sciences, vol. 2015, Article ID 417327, 10 pages, 2015.

[39] A. de Médicis and P. Leroux, "Generalized Stirling numbers, convolution formulae and p; q-analogues," Canadian Journal of Mathematics, vol. 47, no. 3, pp. 474-499, 1995.

[40] B. S. El-Desouky, "The multiparameter noncentral Stirling numbers," The Fibonacci Quarterly, vol. 32, pp. 218-225, 1994.

[41] R. B. Corcino and M. M. Mangontarum, "On multiparameter $q$-noncentral Stirling and Bell numbers," Ars Combinatoria, vol. 118, pp. 201-220, 2015.
[42] M. M. Mangontarum and J. Katriel, "On $q$-boson operators and $q$-analogues of the $r$-Whitney and $r$-dowling numbers," Journal of Integer Sequences, vol. 18, article 15.9.8, 2015.

[43] M. Arik and D. D. Coon, "Hilbert spaces of analytic functions and generalized coherent states," Journal of Mathematical Physics, vol. 17, no. 4, pp. 524-527, 1976. 


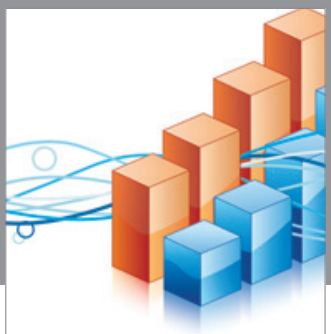

Advances in

Operations Research

vatem alat4

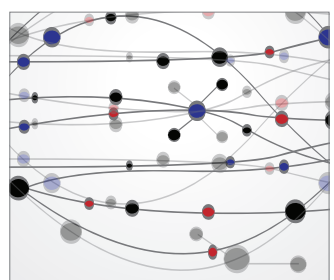

\section{The Scientific} World Journal
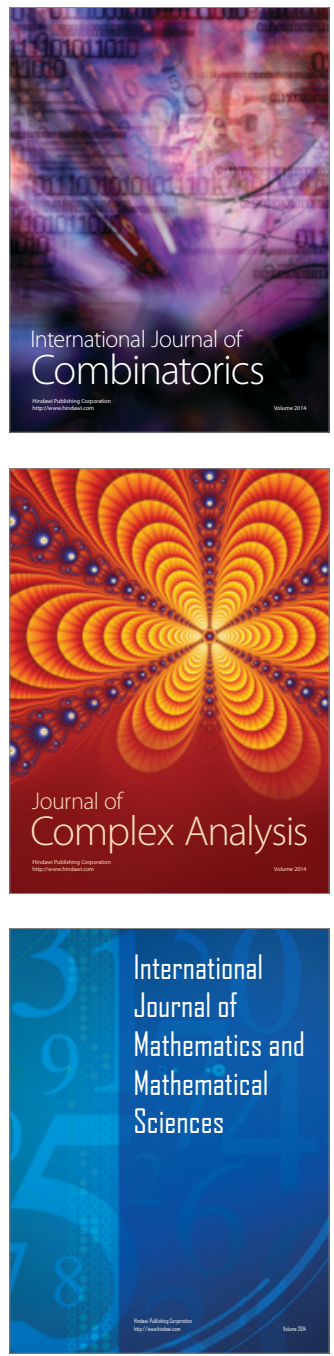
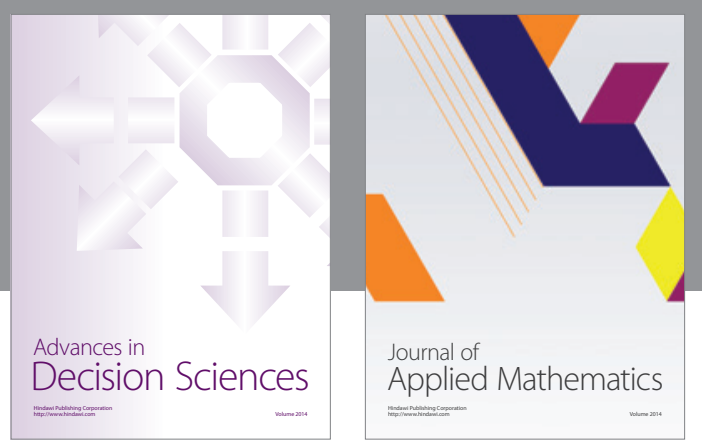

Algebra

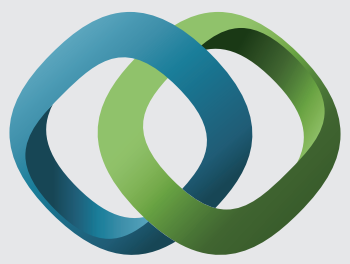

\section{Hindawi}

Submit your manuscripts at

http://www.hindawi.com
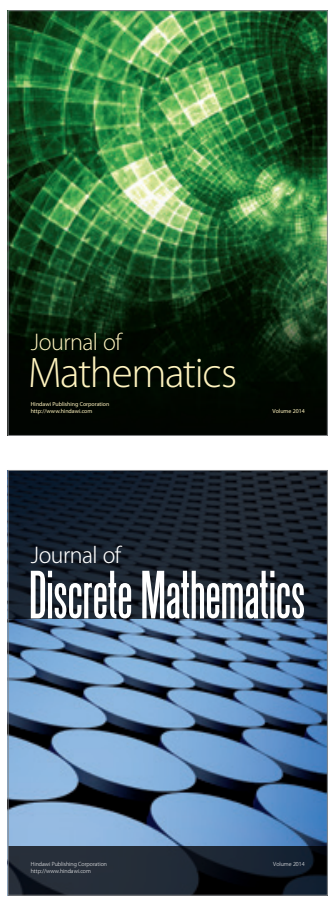

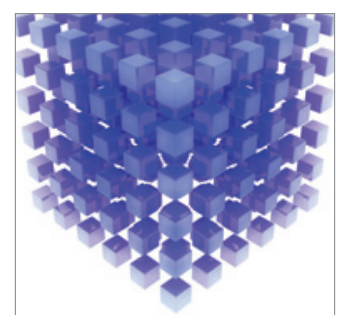

Mathematical Problems in Engineering
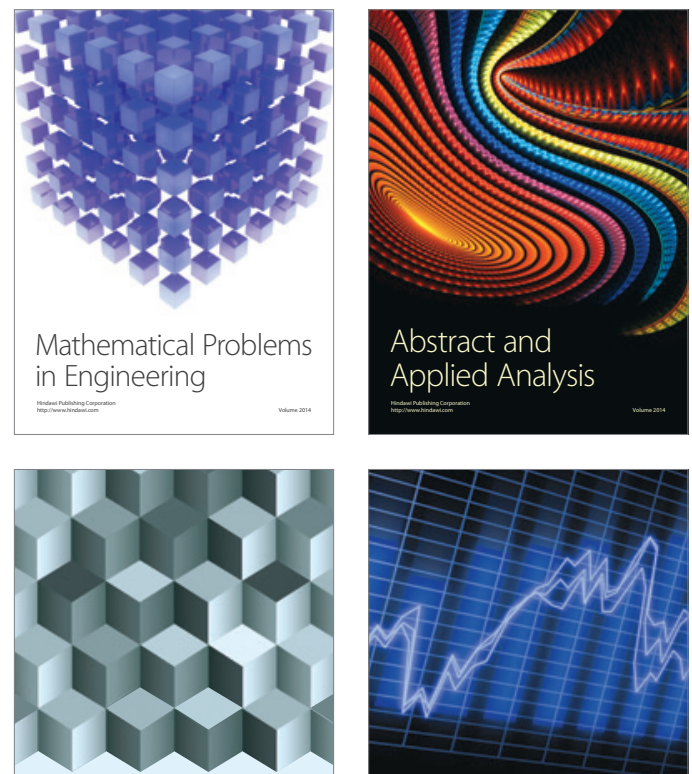

Journal of

Function Spaces

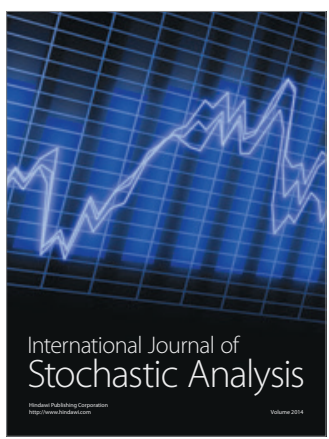

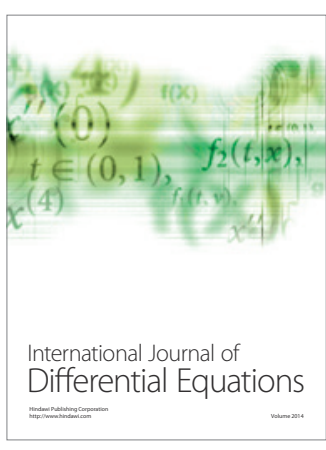
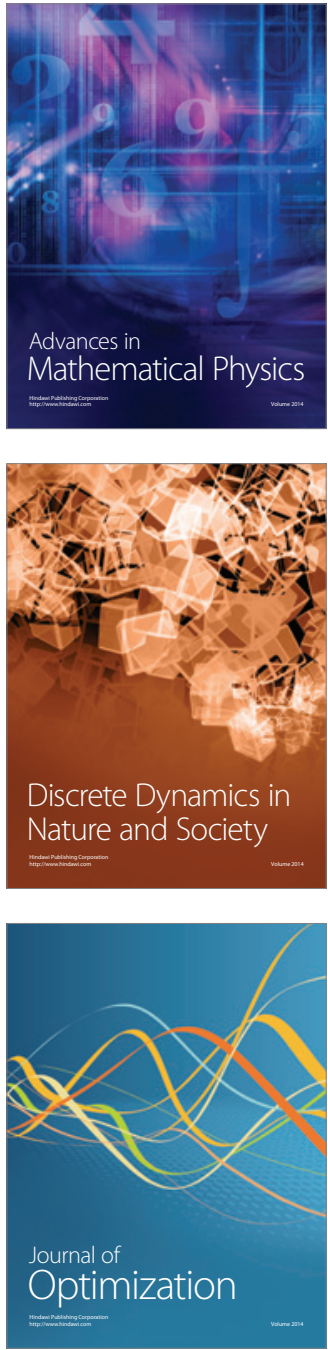